\title{
Are Smaller Nanoparticles Always Better? Understanding the Biological Effect of Size-Dependent Silver Nanoparticle Aggregation Under Biorelevant Conditions
}

\author{
Péter Bélteky $\mathbb{D D}^{1, *}$ \\ Andrea Rónavári ${ }^{1} *$ \\ Dalma Zakupszky' \\ Eszter Boka \\ Nóra lgaz ${ }^{2}$ \\ Bettina Szerencsés ${ }^{3}$ \\ Ilona Pfeiffer ${ }^{3}$ \\ Csaba Vágvölgyi ${ }^{3}$ \\ Mónika Kiricsi (DD ${ }^{2}$ \\ Zoltán Kónya $\mathbb{D}^{1,4}$
}

'Department of Applied and Environmental Chemistry, Faculty of Science and Informatics, University of Szeged, Szeged, Hungary; ${ }^{2}$ Department of Biochemistry and Molecular Biology, Faculty of Science and Informatics, University of Szeged, Szeged, Hungary;

${ }^{3}$ Department of Microbiology, Faculty of Science and Informatics, University of Szeged, Szeged, Hungary; ${ }^{4}$ MTA-SZTE Reaction Kinetics and Surface Chemistry Research Group, Szeged, Hungary

*These authors contributed equally to this work

\begin{abstract}
Purpose: Silver nanoparticles (AgNPs) are one of the most commonly investigated nanomaterials, especially due to their biomedical applications. However, their excellent cytotoxic and antimicrobial activity is often compromised in biological media due to nanoparticle aggregation. In this work, the aggregation behavior and the related biological activity of three different samples of citrate capped silver nanoparticles, with mean diameters of 10, 20, and $50 \mathrm{~nm}$, respectively, were examined.
\end{abstract}

Methods: Following nanoparticle synthesis and characterization with transmission electron microscopy, their aggregation behavior under various $\mathrm{pH}$ values, $\mathrm{NaCl}$, glucose, and glutamine concentrations, furthermore in cell culture medium components such as Dulbecco's Modified Eagle's Medium and fetal bovine serum, was assessed through dynamic light scattering and ultraviolet-visible spectroscopy.

Results: The results indicated that acidic $\mathrm{pH}$ and physiological electrolyte content universally induce micron-scale aggregation, which can be mediated by biomolecular corona formation. Remarkably, larger particles demonstrated higher resistance against external influences than smaller counterparts. In vitro cytotoxicity and antimicrobial assays were performed by treating cells with nanoparticulate aggregates in differing stages of aggregation.

Conclusion: Our results revealed a profound association between colloidal stability and toxicity of AgNPs, as extreme aggregation led to the complete loss of biological activity. The higher degree of aggregation resistance observed for larger particles had a significant impact on the in vitro toxicity, since such samples retained more of their activity against microbes and mammalian cells. These findings lead to the conclusion that aiming for the smallest possible nanoparticles might not be the best course of action, despite the general standpoint of the relevant literature.

Keywords: seed-mediated growth, colloidal stability, size-dependent aggregation behavior, aggregation compromised toxicity

\section{Introduction}

With the increasing demand and production of nanomaterials, more and more attention is paid to their biosafety, or alternatively, biological activity. Silver nanoparticles (AgNPs) are one of the most commonly synthesized, investigated, and utilized representatives of such materials, due to their excellent catalytic,
Correspondence: Zoltán Kónya

Environmental Chemistry, Faculty of

Science and Informatics, University of

Szeged, Rerrich Square I, Szeged, H-6720,

Hungary

Tel +36 62544620

Email konya@chem.u-szeged.hu 
optical, and biological properties. ${ }^{1}$ It is generally acknowledged that the unique properties of nanosized materials (including AgNPs) are attributed primarily to their large specific surface area, therefore, the question inevitably arises whether any process that influences this key feature, such as particle size, surface coating, or aggregation, would severely compromise the nanoparticle characteristics crucial for specific applications.

The effects of particle size and stabilizing agents are topics that are already relatively well documented in the literature. For instance, it is a commonly accepted notion, that smaller nanoparticles are more toxic than their larger counterparts. ${ }^{2}$ In agreement with the general literature, our previous studies have demonstrated the size-dependent activity of nanosilver on mammalian cells and microbes alike. ${ }^{3-5}$ Surface coating is another attribute that has widespread effects on the properties of nanomaterials. The same nanomaterial might possess widely different physical, chemical, and biological characteristics just by adding, or modifying stabilizing agents on its surface. The application of the capping agents is most routinely performed as part of nanoparticle synthesis. For instance, citrate capped silver nanoparticles, which are one of the most relevant AgNPs in research, are synthesized through the reduction of a silver salt in a solution of the selected stabilizing agent acting as the reaction medium. ${ }^{6}$ Citrate can be readily utilized due to its low cost, availability, biocompatibility and its strong affinity to silver, which can manifest in a variety of proposed interactions, from reversible surface adsorption, to ionic intercations. ${ }^{7,8} \mathrm{Next}$ to small molecules and polyatomic ions such as citrate, polymers, polyelectrolytes and biological agents are also commonly used to stabilize and uniquely functionalize nanosilver. $^{9-12}$

While the possibilities to modify nanoparticle activity through intentional surface capping is a very interesting field, the main role of such surface coatings is rather trivial, to provide colloidal stability for nanoparticulate systems. The large specific surface area of nanomaterials generates large surface energy, which opposes the thermodynamic aptitude of systems to reach their energy minimum. ${ }^{13}$ Without proper stabilization, this leads to the aggregation of nanomaterials. Aggregation is the formation of particle assemblies in various shapes and sizes that occurs when dispersed particles meet and the present thermodynamic interactions allow the particles to attach to one another. Stabilizing agents are therefore utilized to prevent aggregation by introducing large enough repulsive forces among particles, to counteract their thermodynamic attraction. $^{14}$

Although the topics of particle size and surface capping have been thoroughly explored in the context of their modulatory effect on nanoparticle triggered biological activities, particle aggregation is a largely neglected area. Thorough investigations tackling the colloidal stability of nanoparticles in biologically relevant conditions can scarcely be found. ${ }^{10,15-17}$ Furthermore, such contributions are especially rare, where aggregation related toxicity is also investigated, even though it can lead to adverse reactions, for example, vascular thrombosis, or loss of desired properties, eg, their toxicity, as illustrated in Figure $1 .{ }^{18,19}$ In fact, one of the few known mechanisms of silver nanoparticle resistance is associated with aggregation, as certain E. coli and $P$. aeruginosa strains were reported to reduce their nanosilver susceptibility by the expression of the protein flagellin, which has a high binding affinity toward silver, hence inducing aggregation. ${ }^{20}$

There are several distinct mechanisms related to silver nanoparticle toxicity, and aggregation affects all of them. The most discussed method of AgNP biological activity, sometimes referred to as the "Trojan horse" mechanism, considers AgNPs mainly as $\mathrm{Ag}^{+}$carriers. ${ }^{1,21}$ The Trojan horse mechanism can ensure the substantial increase of local $\mathrm{Ag}^{+}$concentration, which can lead to ROS generation, and membrane depolarization. ${ }^{22-24}$ Aggregation may affect $\mathrm{Ag}^{+}$release and thus, toxicity, as it reduces the effective active surface where the oxidative dissolution of silver ions is a possibility. However, AgNPs do not show toxicity simply by ion release, a number of size and morphology dependent interactions must be considered, where the size and shape of the nanoparticle surface is the defining feature. ${ }^{4,25}$ The collection of these mechanisms can be classified as "Inductive toxicity mechanisms", underlying numerous mitochondrial and surface membrane reactions that may damage organelles and lead to cell death. ${ }^{25-27}$ As the formation of aggregates naturally affects the size and shape of the silver-containing objects as identified by living systems, these interactions may also be compromised.

In our previous paper addressing silver nanoparticle aggregation, we demonstrated an efficient screening procedure consisting of chemical and in vitro biological experiments for investigating the issue. ${ }^{19}$ Dynamic light scattering (DLS) is a technique of choice for these types of examinations, since materials are able to scatter photons, which have wavelengths comparable to their particle sizes. 


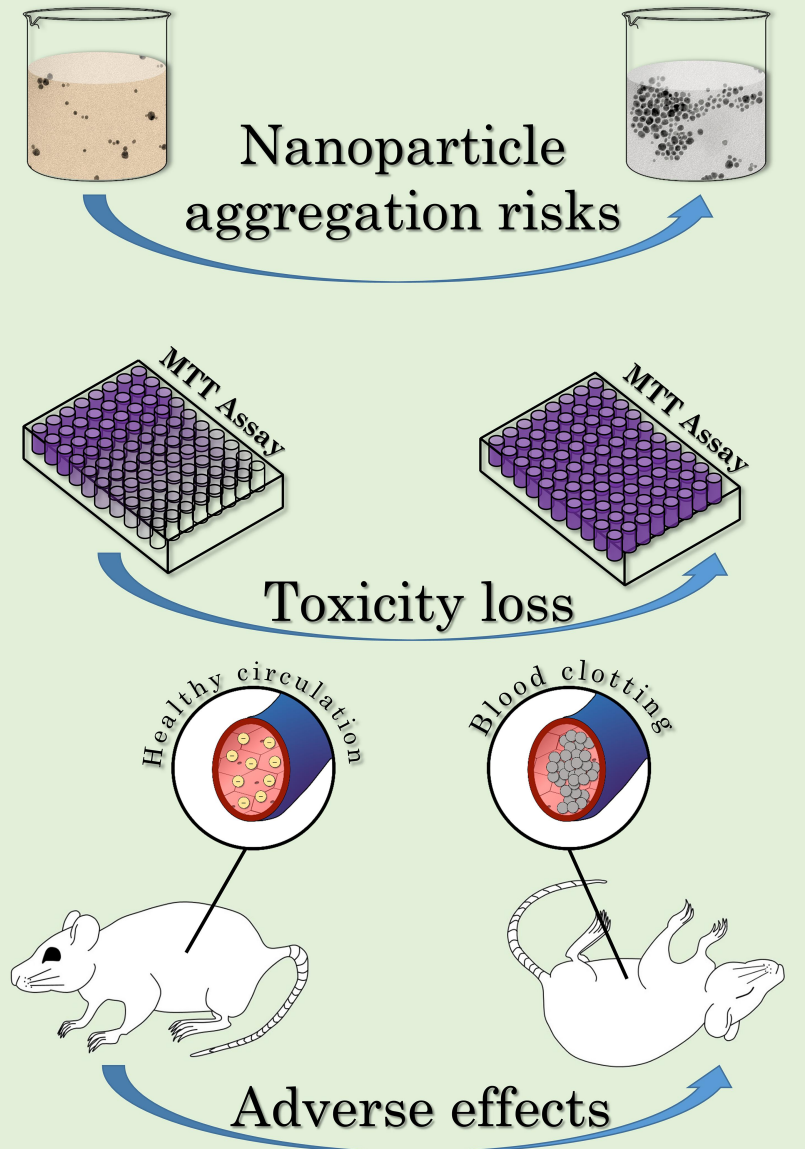

Figure I Illustration of the risks of nanoparticle aggregation.

As the speed of the Brownian motion of a particle in liquid media is size-dependent, the changes in scattered light intensity can be used to determine the average hydrodynamic diameter (Z-average) of liquid samples. ${ }^{28}$ Additionally, by applying voltage to the samples, the zeta potential ( $\zeta$-potential) of the nanoparticles can be measured similarly to Z-average. ${ }^{13,28}$ If the absolute value of $\zeta$-potential is high enough (according to common guidelines $> \pm 30 \mathrm{mV}$ ), it generates strong enough electrostatic repulsion between particles, to counteract aggregation. Characteristic surface plasmon resonance (SPR) is a unique optical phenomenon mostly attributed to noble metal nanoparticles (mainly Au and Ag). ${ }^{29}$ Based on the electron oscillations (surface plasmons) of such materials in the nanoscale, spherical AgNPs are known to possess a characteristic UV-Vis absorbance peak around $400 \mathrm{~nm} .{ }^{30}$ The intensity and wavelength shifts of the particles were used to compliment the DLS results, as nanoparticle aggregation and biomolecule surface adsorption could be detected with this method.
With the resulting information, cell viability (MTT) and antimicrobial assays were performed in a way where AgNP toxicity was described as the function of aggregation grade instead of (the most commonly used factor) nanoparticle concentration. This unique approach enabled us to show the profound importance of aggregation grade in biological activity since for instance, citrate capped AgNPs completely lost their biological activity due to aggregation within a few hours. ${ }^{19}$

In this present work, we aimed to substantially extend our previous contributions regarding biorelevant colloidal stability and its consequences on biological activity, by investigating the influence of nanoparticle size on nanoparticle aggregation, which is unarguably a much more high-profile angle of nanoparticle research and a constant, defining characteristic throughout biologically relevant applications. ${ }^{31}$ To investigate this issue, citrate capped AgNPs were produced in three distinct size ranges $-10,20$, and $50 \mathrm{~nm}$ - using the seedmediated growth method. ${ }^{6,32}$ As one of the most broadly and routinely utilized nanomaterials for medical applications, differently-sized citrate-capped AgNPs were selected to perform this study on the possible sizedependence of the aggregation-related biological performance of nanosilver. Following the synthesis of differently sized AgNPs, we characterized the produced samples by transmission electron microscopy (TEM), then the particles were examined using the before mentioned screening procedure. The size-dependent aggregation behavior under various $\mathrm{pH}$ values, $\mathrm{NaCl}$, glucose, and glutamine concentrations, furthermore, in the presence of the in vitro cell culture Dulbecco's Modified Eagle's Medium (DMEM) and fetal bovine serum (FBS) was assessed and their cytotoxic features under aggregated circumstances were determined. Scientific consensus says that smaller particles are more preferable in general; our investigation provides a chemical and biological platform to judge whether this really is the case.

\section{Materials and Methods}

\section{Nanoparticle Synthesis}

All chemicals were purchased from Sigma-Aldrich (Saint Louis, MO, USA).

Silver nanoparticles in three different size ranges were prepared by the seed-mediated growth approach proposed by Wan et al with slight adjustments. ${ }^{6}$ The method is based on chemical reduction using silver nitrate $\left(\mathrm{AgNO}_{3}\right)$ as 
a silver source, sodium borohydride $\left(\mathrm{NaBH}_{4}\right)$ as reductant, and sodium citrate as the stabilizing agent. Firstly, $75 \mathrm{~mL}$ of $9 \mathrm{mM}$ aqueous citrate solution was prepared from sodium citrate dihydrate $\left(\mathrm{Na}_{3} \mathrm{C}_{6} \mathrm{H}_{5} \mathrm{O}_{7} \times 2 \mathrm{H}_{2} \mathrm{O}\right)$ and heated to $70^{\circ} \mathrm{C}$. Then, $2 \mathrm{~mL} 1 \% \mathrm{w} / \mathrm{v} \mathrm{AgNO}_{3}$ solution was added to the reaction medium, followed by a freshly prepared solution of sodium borohydride $(2 \mathrm{~mL} 0.1 \% \mathrm{w} / \mathrm{v})$ which was poured dropwise into the mixture. The resulting yellowish-brown suspension was kept at $70^{\circ} \mathrm{C}$ under vigorous stirring for 1 hour and afterward was left to cool to room temperature. The resulting sample (from now on referred to as AgNP-I) was used as the basis for seed-mediated growth in the next synthesis step.

To synthesize the medium sized particle suspension (denoted as AgNP-II), $90 \mathrm{~mL}$ of $7.6 \mathrm{mM}$ citrate solution was heated to $80^{\circ} \mathrm{C}$, which was mixed with $10 \mathrm{~mL}$ of AgNP-I followed by $2 \mathrm{~mL}$ of $1 \% \mathrm{w} / \mathrm{v} \mathrm{AgNO}_{3}$ solution under vigorous mechanical stirring for 1 hour, then the sample was cooled to room temperature.

For the largest particles (AgNP-III), the same growth procedure was repeated, but in this case, $10 \mathrm{~mL}$ AgNP-II was used as seed suspension. After the samples reached room temperature, their nominal $\mathrm{Ag}$ concentrations based on total $\mathrm{AgNO}_{3}$ content were set to $150 \mathrm{ppm}$ by adding or evaporating extra solvent on $40^{\circ} \mathrm{C}$, and ultimately they were stored at $4{ }^{\circ} \mathrm{C}$ until further use.

\section{Nanoparticle Characterization}

The morphological characteristics of the nanoparticles were examined and their electron diffraction (ED) patterns were captured using an FEI Tecnai $\mathrm{G}^{2} 20 \mathrm{X}$-Twin transmission electron microscope (TEM) with $200 \mathrm{kV}$ accelerating voltage (FEI Corporate Headquarters, Hillsboro, OR, USA). At least 15 representative images ( 750 particles) were evaluated using the ImageJ software package and the resulting histograms (and all graphs throughout the research) were created in OriginPro 2018 (OriginLab, Northampton, MA, USA). ${ }^{33,34}$

The average hydrodynamic diameter (Z-average), zeta potential ( $\zeta$-potential), and characteristic surface plasmon resonance (SPR) of the samples were measured to illustrate their initial colloidal properties. The average hydrodynamic diameter and zeta potential of the samples were measured at $37 \pm 0.1{ }^{\circ} \mathrm{C}$ by a Malvern Zetasizer Nano ZS instrument (Malvern Instruments, Malvern, UK) using disposable folded capillary cells. Characteristic SPR properties were obtained from the UV-Vis absorbance spectra of the samples within the range of $250-800 \mathrm{~nm}$ using an Ocean Optics 355 DH-2000-BAL UV-Vis spectrophotometer (Halma PLC, Largo, FL, USA).

\section{Aggregation Behavior Measurements}

Throughout the experiments, three different measurement types associated with colloidal stability were performed simultaneously. The average hydrodynamic diameter (Z-average) and zeta potential ( $\zeta$-potential) of the particles were measured using DLS, as Z-average correlates with the mean size of nanoparticle aggregates and zeta potential shows if the electrostatic repulsion within the system is strong enough to counteract the van der Waals attraction between nanoparticles. The measurements were performed in triplicate, the standard deviation of Z-average and $\zeta$ potential was calculated by the Zetasizer software. The characteristic SPR spectra of the particles were assessed via UVVis spectroscopy, as peak intensity changes and wavelength shifts can indicate aggregation and surface interactions. ${ }^{29,35}$ Surface plasmon resonance in noble metals is so influential in fact, that it has led to new analytical approaches for biomolecules. $^{29,36,37}$ The concentration of AgNPs in the experimental mixtures was around $10 \mathrm{ppm}$, with the aim of setting the intensity of their starting SPR absorption maximum to 1 . The experiments were carried out in a timedependent manner at $0 ; 1.5 ; 3 ; 6 ; 12$ and 24 hours under various biorelevant conditions. More details describing the experiments can be seen in our previous work. ${ }^{19}$ Briefly, various $\mathrm{pH}$ values $(3 ; 5 ; 7.2$ and 9$)$, different sodium chloride $(10 \mathrm{mM} ; 50 \mathrm{mM} ; 150 \mathrm{mM})$, glucose $(3.9 \mathrm{mM} ; 6.7 \mathrm{mM})$ and glutamine $(4 \mathrm{mM})$ concentrations, moreover Dulbecco's Modified Eagle Medium (DMEM) and fetal bovine serum (FBS) (in water and DMEM) were prepared as model systems, and their effect on the aggregation behavior of the synthesized silver nanoparticles was investigated. The values of $\mathrm{pH}, \mathrm{NaCl}$, glucose, and glutamine were assessed from physiological concentrations, while the amount of DMEM and FBS were identical to the levels used throughout the in vitro experiments. ${ }^{38-42}$ All measurements were performed at $\mathrm{pH} 7.2$ and $37^{\circ} \mathrm{C}$ with a constant background salt concentration of $10 \mathrm{mM} \mathrm{NaCl}$ to negate any long-distance particle interactions (with the exceptions of certain $\mathrm{pH}-$ and $\mathrm{NaCl}$ related experiments where these attributes were the investigated variables). ${ }^{28}$ The list of the various conditions is summarized in Table 1, where the experiment marked with $\dagger$ was used as a reference and corresponds to a sample containing $10 \mathrm{mM} \mathrm{NaCl}$ on $\mathrm{pH}$ 7.2. 
Table I List of Applied Biorelevant Conditions

\begin{tabular}{|l|c|}
\hline Condition & Value(s) \\
\hline $\mathrm{pH}$ & $3 ; 5 ; 7.2^{\dagger} ; 9$ \\
$\mathrm{NaCl}$ concentration (mM) & $10 ; 50 ; 150$ \\
Glucose concentration (mM) & $3.9 ; 6.7$ \\
Glutamine concentration (mM) & 4 \\
DMEM (v/v\%) & 45 \\
FBS (v/v\%, both in water and DMEM mixture) & 5 \\
\hline
\end{tabular}

Notes: ${ }^{\dagger}$ Represents the reference point for the measurements; all samples were measured at $37^{\circ} \mathrm{C}, \mathrm{pH} \sim 7.2$ with $10 \mathrm{mM} \mathrm{NaCl}$ electrolyte background unless otherwise stated.

\section{Cytotoxicity Analysis}

Human prostate cancer cell line (DU145) and immortalized human keratinocyte cells (HaCaT) were obtained from ATCC (Manassas, VA, USA). Cells were routinely cultivated in Dulbecco's Minimum Essential Medium Eagle (DMEM) containing $4.5 \mathrm{~g} / \mathrm{L}$ glucose (SigmaAldrich, Saint Louis, MO, USA), supplemented with 10\% FBS, 2 mM L-glutamine, $0.01 \%$ streptomycin, and $0.005 \%$ penicillin (Sigma-Aldrich, Saint Louis, MO, USA). Cells were cultured in a $37^{\circ} \mathrm{C}$ incubator at $5 \%$ $\mathrm{CO}_{2}$ in $95 \%$ humidity.

To explore the changes in AgNP cytotoxicity caused by particle aggregation in a time-dependent manner, a twostep MTT assay was carried out. First, the viability of both cell types was measured after AgNP-I, AgNP-II, and AgNP-III treatments. For this, both types of cells were seeded into 96-well plates in 10,000 cells/well density and treated on the following day with silver nanoparticles of three different sizes in increasing concentrations. After $24 \mathrm{~h}$ treatments, cells were washed with PBS and incubated for an hour at $37^{\circ} \mathrm{C}$ with $0.5 \mathrm{mg} / \mathrm{mL}$ MTT reagent (SERVA, Heidelberg, Germany) diluted in culture medium. Formazan crystals were solubilized in DMSO (Sigma-Aldrich, Saint Louis, MO, USA) and absorption was measured at $570 \mathrm{~nm}$ using a Synergy HTX plate reader (BioTek-Hungary, Budapest, Hungary). Absorption values of the untreated control samples were considered as $100 \%$ viability. Experiments were performed at least three times using four independent biological replicates. $\mathrm{IC}_{50}$ was calculated by the dose-response curve based on the viability results.

Thereafter, in the second step, different silver nanoparticle aggregation states were created by incubating the particles with $150 \mathrm{mM} \mathrm{NaCl}$ for different time periods $(0,1.5,3$, 6,12 , and 24 hours) prior to cell treatments. Subsequently, the same MTT assay was performed as described previously, to assess the changes in cell viability affected by particle aggregation. The final results were assessed using GraphPad Prism 7, the statistical significance of the experiments was calculated through unpaired $t$-tests and their levels were marked as $*(\mathrm{p} \leq 0.05), * *(\mathrm{p} \leq 0.01), * * *(\mathrm{p} \leq 0.001)$, and $* * * *(\mathrm{p} \leq 0.0001)$.

\section{Antimicrobial Assays}

The antimicrobial susceptibility test was carried out using the three differently sized silver nanoparticles (AgNP-I, AgNP-II, and AgNP-III) on Cryptococcus neoformans IFM 5844 (IFM; Research Center for Pathogenic Fungi and Microbial Toxicoses, Chiba University), Bacillus megaterium SZMC 6031 (SZMC: Szeged Microbiology Collection), and Escherichia coli SZMC 0582 in RPMI 1640 medium (Sigma-Aldrich Co.). To evaluate the changes in antimicrobial activities caused by particle aggregation, first, their minimal inhibitory concentration (MIC) was determined with the microdilution method in 96-well microplates. To $50 \mu \mathrm{L}$ of standardized cell suspension $\left(5 \times 10^{4}\right.$ cell $/ \mathrm{mL}$ in RPMI 1640 medium), $50 \mu \mathrm{L}$ silver nanoparticle suspension was added in serially two-folddiluted concentrations (in the range of 0 and $75 \mathrm{ppm}$ in the previously described medium, namely the control samples contained $50 \mu \mathrm{L}$ cell suspension and $50 \mu \mathrm{L}$ medium without nanoparticles). Thereafter, the plates were incubated at $30^{\circ} \mathrm{C}$ for 48 hours and the optical density of the cultures was detected at $620 \mathrm{~nm}$ using SPECTROstar Nano plate reader (BMG LabTech, Offenburg, Germany). The experiments were carried out three times in triplicate.

The effect of aggregation on antimicrobial activity was examined on the previously mentioned strains with the same procedure as described previously, except that at this time $50 \mu \mathrm{L}$ individual aggregated nanoparticle samples were used. The different silver nanoparticle aggregation states were created by incubating the particles with $150 \mathrm{mM} \mathrm{NaCl}$ for different time periods $(0,1.5,3,6,12$, and 24 hours) before cell treatments. Suspensions supplemented with $50 \mu \mathrm{L}$ RPMI 1640 medium were used as growth control, while for control of toxicity, suspensions with non-aggregated nanoparticles were used. The experiments were carried out three times in triplicate. The final results were once again assessed using GraphPad Prism 7, with the same statistical analysis used for the MTT assays.

\section{Results and Discussion}

The aggregation grade of the smallest particles (AgNP-I) had already been characterized and the results were 
partially published in our previous work, but for the purpose of a better comparison, a thorough screening was performed on all of the particles, the experimental data of AgNPs in all three sizes have been gathered and discussed in the following sections. ${ }^{19}$

\section{Silver Nanoparticle Morphology and Characteristics}

The measurements performed by TEM, UV-Vis, and DLS verified the successful synthesis of all AgNP samples (Figure 2A-D). The smallest particles (AgNP-I) showed uniform spherical morphology with an average diameter around $10 \mathrm{~nm}$ according to the first row of Figure 2. The seed-mediated growth method also provided AgNP-II and AgNP-III of distinct size ranges with mean particle diameters around $20 \mathrm{~nm}$ and $50 \mathrm{~nm}$ respectively. According to the standard deviation of the particle distributions, the size of the three samples had no overlap, which is important for their comparative analysis. The sphericity of the particles was assumed by comparing the average aspect and thinness ratios of the TEM based 2D projections of the particles, evaluated by the Shape Filter plugin of ImageJ (Figure 2E). ${ }^{43}$ According to the shape analysis of the particles, while their aspect ratio (larger side/shorter side of the minimum bounding rectangle) was unaffected by particle growth, their thinness ratio (measured area/theoretical area of the corresponding perfect circle) gradually decreased, resulting in more and more polyhedric particles, somewhat distancing themselves from the theoretical perfect circle, which corresponds with a thinness ratio of 1 .

Although the cyclical nature of the growth method affected particle shape somewhat which led to lesser sphericity in larger AgNPs, all three samples remained quasi-spherical, furthermore the crystallinity of the nanoparticles remained unaffected as evidenced by the electron diffraction patterns in Figure 2B. The prominent diffraction rings - that can be associated with the (111), (220), (200), and (311) miller indices of silver - are in good agreement with the scientific literature and with our previous contributions as well. 9,19,44 The fragmentation of the Debye-Scherrer rings of AgNP-II and AgNP-III is due to
A
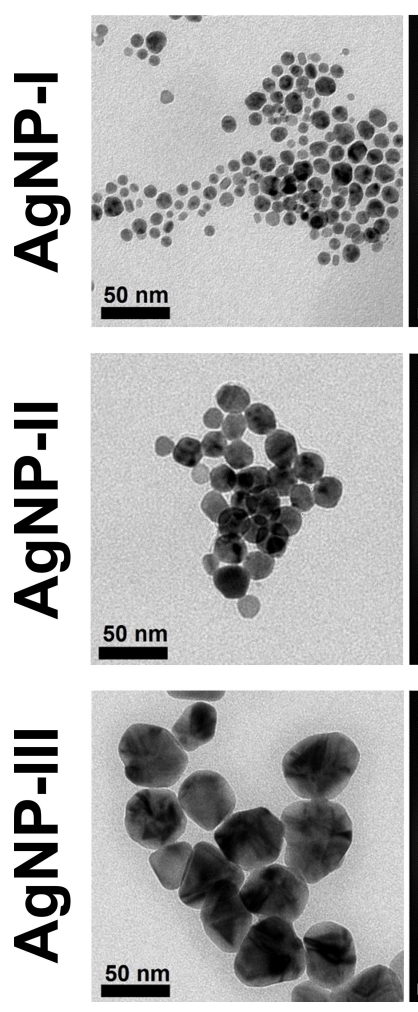

B
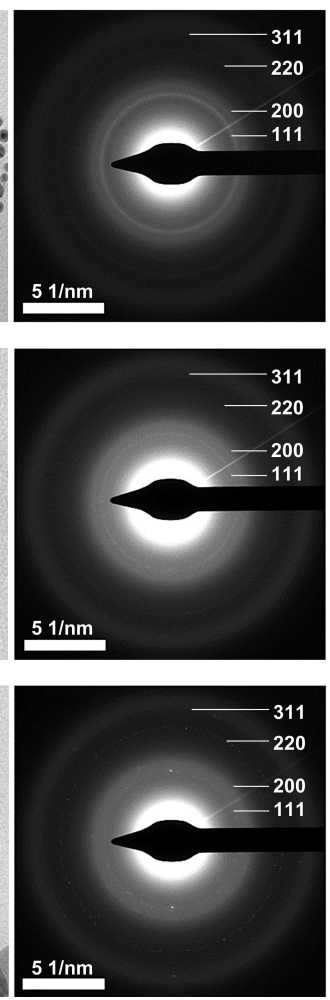

C

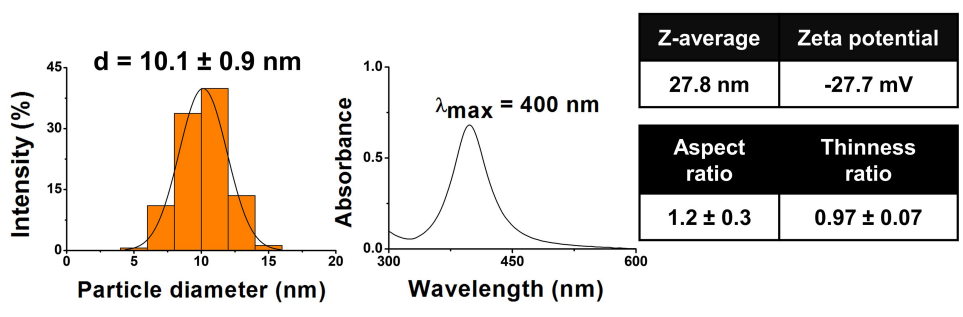

D

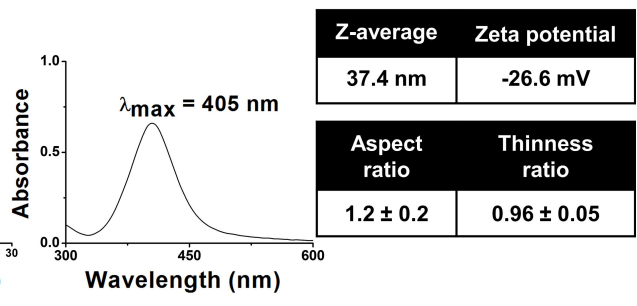

Figure 2 Transmission electron microscope (TEM) images (A), electron diffraction (ED) patterns (B), size distribution histograms (C), characteristic ultraviolet-visible (UVVis) light absorbance spectra (D), furthermore average hydrodynamic diameter (Z-average), zeta potential, aspect ratio and thinness ratio (E) of citrate capped silver nanoparticles with three distinct size ranges: $10 \mathrm{~nm}$ for AgNP-I (top row), $20 \mathrm{~nm}$ for AgNP-II (middle row), and $50 \mathrm{~nm}$ for AgNP-III (bottom row). 
the fact that the ED images were captured with the same magnification, thus with the increase of particle size, the number of diffracting particles per area decreased.

Nanoparticle size and shape are known to affect biological activity., ${ }^{3,45}$ The shape-dependent catalytic and biological activity is explained by the fact that different shapes tend to proliferate certain crystal facets (with distinct miller indices), and these facets have varying activity. ${ }^{45,46}$ Since the as-prepared particles provided analogous ED results corresponding to very similar crystalline characteristics, the assumption could be made that throughout our subsequent colloidal stability and biological activity experiments, any observed discrepancy should be ascribed to nanoparticle size, and not to shape-related attributes.

The UV-Vis results summarized in Figure 2D further underlined the overwhelmingly spherical nature of the synthesized AgNPs, as the SPR peaks of all three samples were around $400 \mathrm{~nm}$, which is a characteristic value for spherical silver nanoparticles. ${ }^{29,30}$ The captured spectra also verified the successful seed-mediated growth of nanosilver, as with increasing particle size, the wavelengths corresponding to the light absorbance maxima of AgNPII and - more prominently - AgNP-III went through a redshift, in accordance with the literature. 6,29

Regarding the initial colloidal stability of the AgNP systems, the average hydrodynamic diameter and zeta potential of the particles were measured on $\mathrm{pH} 7.2$ using DLS. The results depicted in Figure $2 \mathrm{E}$ indicated that AgNP-III possesses greater colloidal stability compared to AgNP-I or AgNP-II, since commonly used guidelines suggest that for long-term colloidal stability, a zeta potential of $30 \mathrm{mV}$ in absolute value is necessary for sufficient electrostatic repulsion. ${ }^{28}$ This finding was further supported when Z-average values (obtained as the average hydrodynamic diameter of free and aggregated particles) were compared to primary particle diameters obtained by TEM, as the closer the two values are, the milder the degree of aggregation is within the samples. In fact, the Z-average of both AgNP-I and AgNP-II were reasonably higher than their primary TEM-assessed particle diameters, thus these samples were predicted to be more susceptible toward aggregation compared to AgNP-III, where the highly negative zeta potential was accompanied by close-to-size $\mathrm{Z}$-average.

The explanation of this phenomenon can be twofold. One aspect is that throughout all synthesis steps, citrate concentration was kept on a similar level, providing relatively higher amounts of charged surface groups against the decreasing specific surface area of the growing particles. However, according to Levak et al, small molecules like citrate can be easily exchanged by biomolecules on nanoparticle surfaces, in which case colloidal stability will be determined by the resulting biomolecular corona. ${ }^{31}$ As this behavior was observed in our aggregation measurements as well (later discussed in more detail), citrate capping cannot explain the phenomenon on its own.

The other aspect is that particle size is inversely proportional to aggregation tendency in the nanoscale. This is mostly supported by conventional Derjaguin-LandauVerwey-Overbeek (DLVO) approaches, where particle attraction is described as the sum of attractive and repulsive forces between particles. According to He et al, the maximum of the DLVO energy curve decreased with nanoparticle size in hematite nanoparticles, allowing the primary energy minimum to be reached more easily, thus facilitating irreversible aggregation (coagulation). ${ }^{47}$ However, it is speculated that there are other aspects exceeding the limitations of the DLVO theory. Even though van der Waals attraction and electrostatic doublelayer repulsion scale similarly with increasing particle size, a review from Hotze et al proposed that it has a stronger effect on aggregation than what DLVO would allow. ${ }^{14}$ They argued that the surface curvature of nanoparticles can no longer be estimated as flat surfaces, making the mathematical estimations inapplicable, furthermore, with decreased particle diameter, a greater percentage of atoms exist on the surface, leading to electronic structure, surface charge behavior, and surface reactivity changes, that could lead to a charge decrease in the electric double layer, facilitating aggregation.

\section{Aggregation Behavior The Effect of $\mathrm{pH}$}

In comparing the DLS results of AgNP-I, AgNP-II, and AgNP-III in Figure 3, we observed that all three samples showed similar $\mathrm{pH}$ prompted aggregation. Heavily acidic environments $(\mathrm{pH} 3)$ turned the zeta potential of the samples toward $0 \mathrm{mV}$ which led to the particles forming micron-sized aggregates, while basic $\mathrm{pH}$ shifted their zeta potentials toward larger negative values where the particles formed smaller aggregates $(\mathrm{pH} 5$ and 7.2), or remained unaggregated altogether $(\mathrm{pH} 9)$. Some important differences between the different samples were also observed. AgNP-I proved to be the most susceptible toward $\mathrm{pH}$-induced changes of zeta potential throughout 


\section{pH Dependence: DLS}
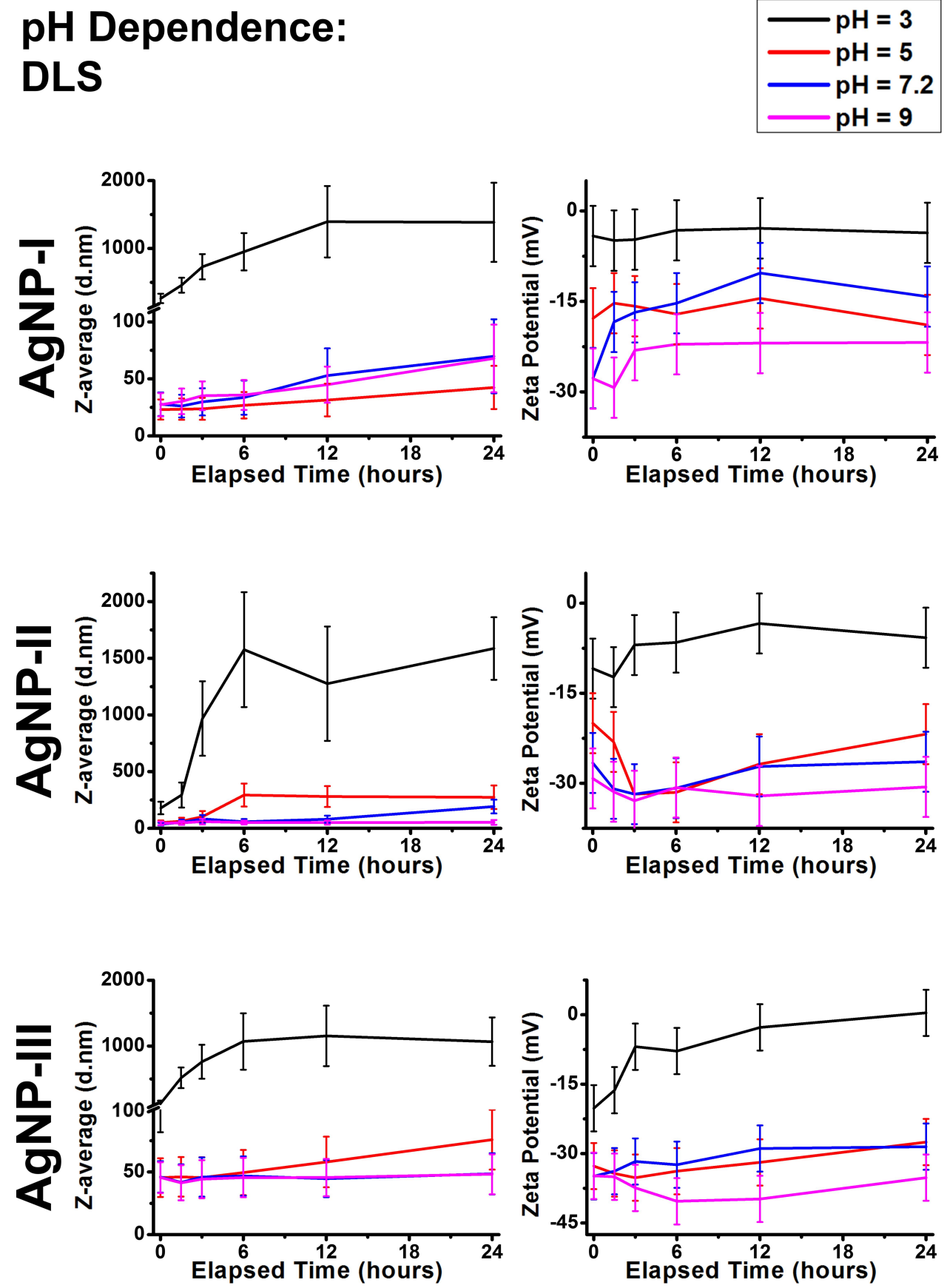

Figure 3 Dynamic light scattering results of citrate capped silver nanoparticle samples with increasing sizes (I0 nm: AgNP-I, $20 \mathrm{~nm}$ : AgNP-II and $50 \mathrm{~nm}$ : AgNP-III) represented as average hydrodynamic diameter (Z-average) (right) and zeta potential (left) changes over 24 hours under conditions with different $\mathrm{pH}$ values.

the experiments, as the zeta potential of these particles already decreased on $\mathrm{pH} 7.2$ compared to $\mathrm{pH}$ 9, while AgNP-II and AgNP-III only demonstrated considerable $\zeta$ changes around $\mathrm{pH}$ 3. Furthermore, AgNP-II showed slower changes and medium zeta potentials, while AgNPIII demonstrated the most moderate behavior of the three, as this system showed the highest zeta absolute values and a slow trending motion indicating that AgNP-III was the most resistant against $\mathrm{pH}$-induced aggregation. These results were in agreement with the average hydrodynamic diameter measurements. Considering their primer particle diameters, AgNP-I demonstrated constant gradual aggregation on all $\mathrm{pH}$ values most likely due to the $10 \mathrm{mM}$ $\mathrm{NaCl}$ background, while AgNP-II and AgNP-III only showed notable aggregation on $\mathrm{pH} 3$. The most interesting difference is that despite its large nanoparticle size, AgNP- 
III formed the smallest aggregates on $\mathrm{pH} 3$ by 24 hours, underlining its resistance against aggregation. By dividing the Z-average of AgNPs on $\mathrm{pH} 3$ after 24 hours by the values of the as-prepared samples, a 50 fold, 42 fold, and 22 fold relative aggregate size increase could be observed respectively for AgNP-I, AgNP-II, and AgNP-III.

The observed $\mathrm{pH}$-dependent aggregation also affected the characteristic surface plasmon resonance (SPR) of the AgNP samples, demonstrated by their UV-Vis spectra. According to Supplementary Figure S1, the aggregation of all three silver nanoparticle suspensions was followed by the intensity decrease and moderate redshift of their SPR peaks. The extent of these changes as the function of $\mathrm{pH}$ was consistent with the degree of aggregation surmised by the DLS results, however, some interesting tendencies were observed. Counterintuitively, the medium sized AgNP-II proved to be the most sensitive toward SPR changes, while the other two samples were less susceptible. In SPR studies, $50 \mathrm{~nm}$ is a theoretical particle size limit which is used to distinguish particles based on their dielectric properties. Particles smaller than $50 \mathrm{~nm}$ (AgNP-I and AgNP-II) can be described as simple dielectric dipoles, while particles at, or above this limit (AgNP-III) have more complex dielectric characteristics, with their resonance bands splitting into multimodal variations. In the case of the two smaller particle samples, where AgNPs can be considered as simple dipoles, plasmons can easily overlap. With increasing particle size, this coupling results in larger plasmons essentially, that could explain the observed greater sensitivity. ${ }^{29}$ For the largest particles, however, where other coupling states can also occur, the simple dipole estimation does not hold, which can explain how AgNP-III demonstrated a decreased propensity for spectral changes. ${ }^{29}$

Within our experimental conditions, $\mathrm{pH}$ proved to have a profound effect on the colloidal stability of citrate capped silver nanoparticles of all sizes. In these systems, stability is provided by negatively charged $-\mathrm{COO}^{-}$groups on the surface of AgNPs. The carboxylate functional groups of the citrate ions undergo protonation in the abundance of $\mathrm{H}^{+}$ions, therefore the resulting carboxyl groups can no longer provide electrostatic repulsion between the particles, as illustrated in the top row of Figure 4. This behavior was clearly observed as all the AgNP samples quickly aggregated on $\mathrm{pH} 3$, but became gradually more and more stable with increasing $\mathrm{pH}$, according to Le Chatelier's principle.

\section{The Effect of $\mathrm{NaCl}$}

Colloidal stability within the differently sized AgNP suspensions was examined under growing salt concentration as well, according to Figure 5. Based on zeta potentials, growing nanoparticle size within these citrate-capped AgNP systems once again provided increasing resistance against the external influence of $\mathrm{NaCl}$. In AgNP-I, $10 \mathrm{mM} \mathrm{NaCl}$ was enough to induce mild aggregation, with $50 \mathrm{mM}$ salt concentration providing very similar results. Whereas, in AgNPII and AgNP-III $10 \mathrm{mM} \mathrm{NaCl}$ did not affect zeta potential considerably as their values stayed around (AgNP-II) or below (AgNP-III) $-30 \mathrm{mV}$. Increasing $\mathrm{NaCl}$ concentration to $50 \mathrm{mM}$, and ultimately to $150 \mathrm{mM} \mathrm{NaCl}$ was sufficient to decrease the absolute values of zeta potential in all the samples substantially, although the larger particles retained more negative charge. These results corresponded with the average hydrodynamic diameter of AgNPs as expected; the Z-average trend lines measured on 10,50 , and $150 \mathrm{mM} \mathrm{NaCl}$ demonstrated distinct, gradually increasing values. Ultimately, micron-sized aggregates were detected in all three of the $150 \mathrm{mM}$ experiments.

The UV-Vis results in Supplementary Figure S2 demonstrated instantaneous and profound SPR declines on 50 and $150 \mathrm{mM} \mathrm{NaCl}$ in all three samples. This can be explained through $\mathrm{DLS}$, as $\mathrm{NaCl}$ based aggregation happened quicker than the $\mathrm{pH}$-dependent experiments, illustrated by the larger differences between the values of the early $(0,1.5$, and $3 \mathrm{~h})$ measurements. Furthermore, increasing salt concentration increases the relative permittivity of the experimental medium as well, which profoundly affects surface plasmon resonance. ${ }^{29}$

The effect of $\mathrm{NaCl}$ is summarized in the middle row of Figure 4. Overall it can be concluded, that increasing sodium chloride concentration had similar effects as increasing acidity, as $\mathrm{Na}^{+}$ions have a tendency to coordinate around carboxylate groups, dampening the negative zeta potential of AgNPs. Furthermore, $150 \mathrm{mM} \mathrm{NaCl}$ generated micron-sized aggregates within all three samples, underlining how physiological electrolyte concentrations are disadvantageous for the colloidal stability of citrate capped AgNPs. These results can be neatly placed in the relevant literature by considering the critical coagulation concentration (CCC) of $\mathrm{NaCl}$ on similar AgNP systems. Huynh et al calculated a CCC of $47.6 \mathrm{mM}$ for $\mathrm{NaCl}$ against citrate capped silver nanoparticles with an average diameter of $71 \mathrm{~nm}$, while El Badawy et al observed $70 \mathrm{mM} \mathrm{CCC}$ for $10 \mathrm{~nm}$ AgNPs with citrate 


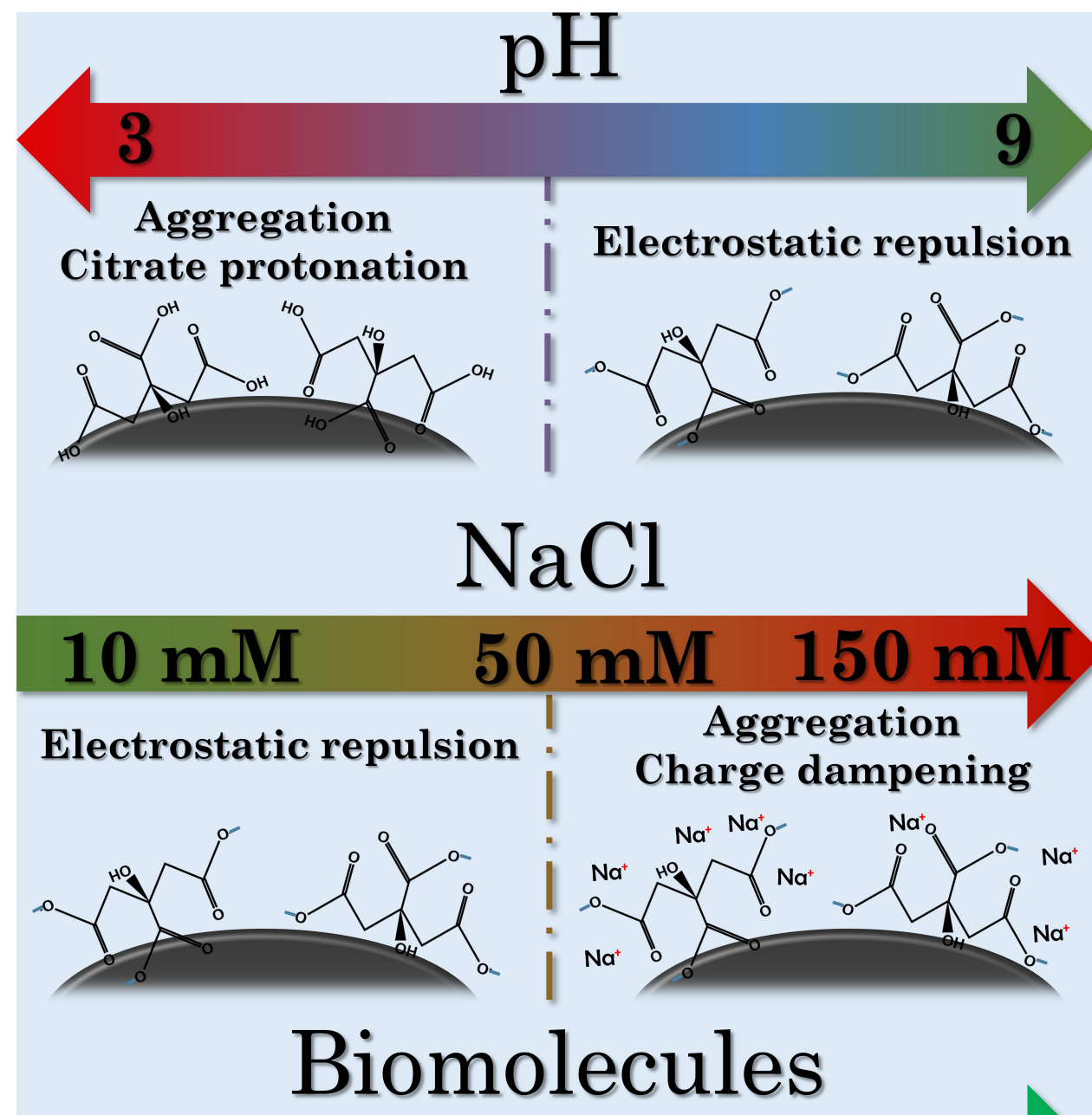

\section{None Physiological amount}

\section{Electrostatic repulsion}

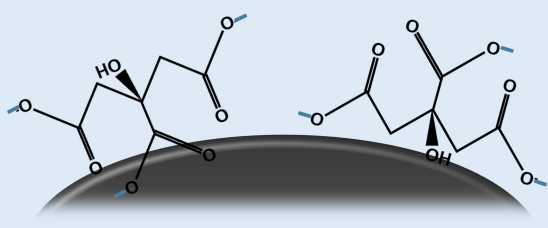

Electrosteric repulsion Surface adsorption

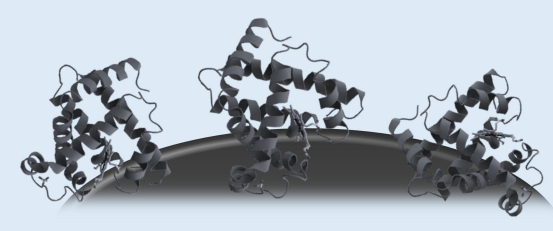

Figure 4 Schematic mechanisms of the aggregation-defining surface interactions under different $\mathrm{pH}$ (top row), $\mathrm{NaCl}$ concentrations (middle row) and biomolecules (bottom row).

coating. ${ }^{10,16}$ Additionally, remarkably high CCC of $\sim 300$ $\mathrm{mM}$ was measured by $\mathrm{He}$ et al, which they contributed to the differences of their synthesis method compared to the before-mentioned publications. ${ }^{48}$ While this current contribution did not aim to perform a comprehensive analysis on such values as our experimental conditions are increasing in complexity throughout the research, the biologically relevant $\mathrm{NaCl}$ concentrations of $50 \mathrm{mM}$ and especially 150 $\mathrm{mM} \mathrm{NaCl}$ seem to be reasonably high to induce coagulation, explaining the robust changes detected.

The Effect of Glucose and Glutamine

The next step of the aggregation experiments was to use simple, yet biologically relevant molecules for modeling 
$\mathrm{NaCl}$ Dependence: DLS
$10 \mathrm{mM} \mathrm{NaCl}$

$50 \mathrm{mM} \mathrm{NaCl}$

$150 \mathrm{mM} \mathrm{NaCl}$
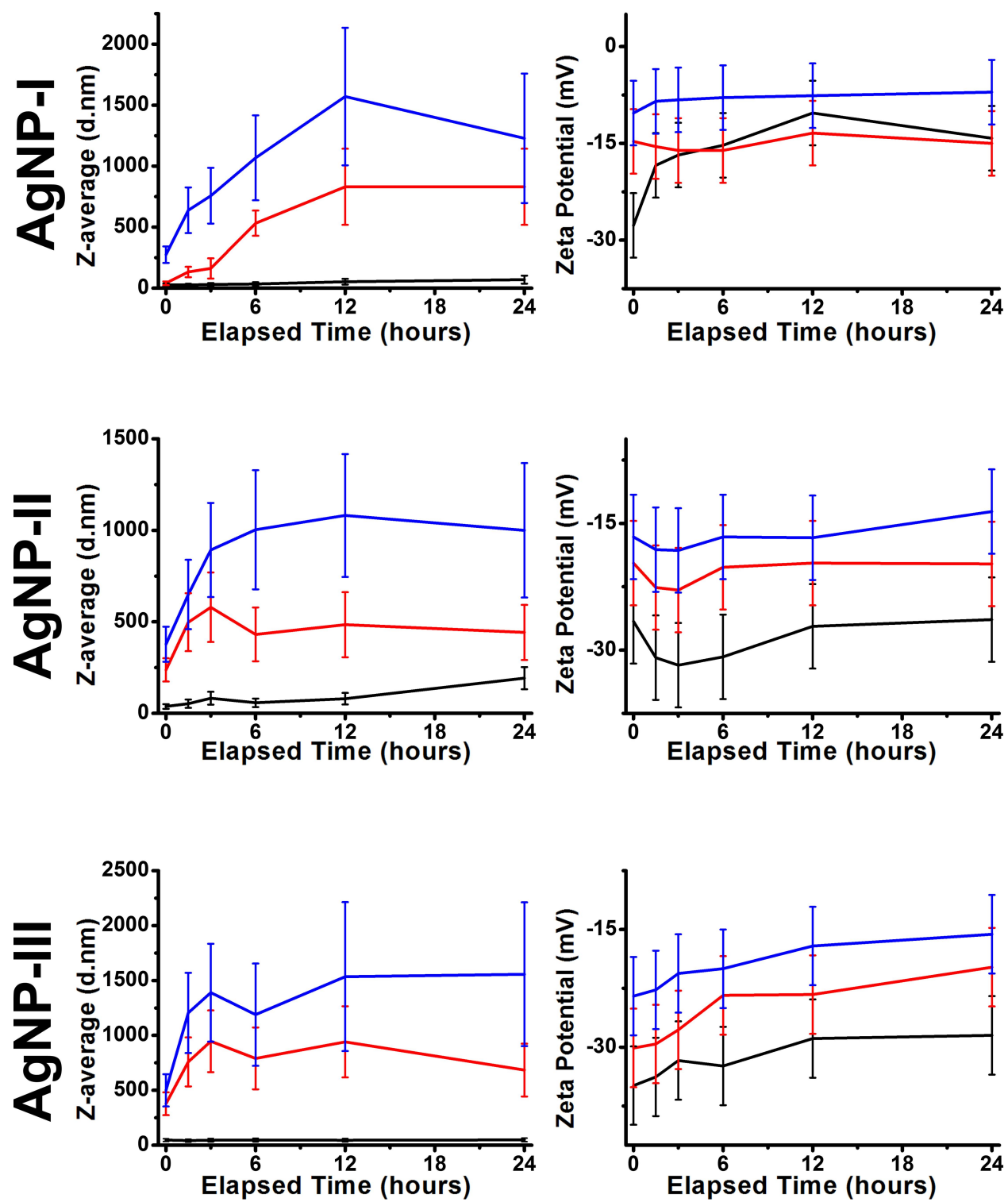

Figure 5 Dynamic light scattering results of citrate capped silver nanoparticle samples with increasing sizes (I0 nm: AgNP-I, $20 \mathrm{~nm}$ : AgNP-II and $50 \mathrm{~nm}$ : AgNP-III) represented as average hydrodynamic diameter (Z-average) (right) and zeta potential (left) changes over 24 hours in different $\mathrm{NaCl}$ concentrations.

nanoparticle-biomolecule interactions. Based on the DLS (Figures 6 and 7) and UV-Vis results (Supplementary $\underline{\text { Figures S3 }}$ and S4), some general conclusions can be asserted. Within our experimental conditions, the investigated molecules glucose and glutamine did not induce aggregation in either of the AgNP systems, as the $\mathrm{Z}$-average trends closely followed the corresponding reference measurements. Despite their presence not affecting aggregation, the experimental results indicated that these molecules were partially adsorbed on the surface of AgNPs. The most prominent results supporting this notion are the observed light absorbance changes. Even though AgNP-I did not demonstrate meaningful wavelength or intensity shifts, more distinct observations could be made measuring the larger particles, most likely due to their before-mentioned larger optical sensitivity. Regardless of 


\section{Glucose Dependence: DLS}
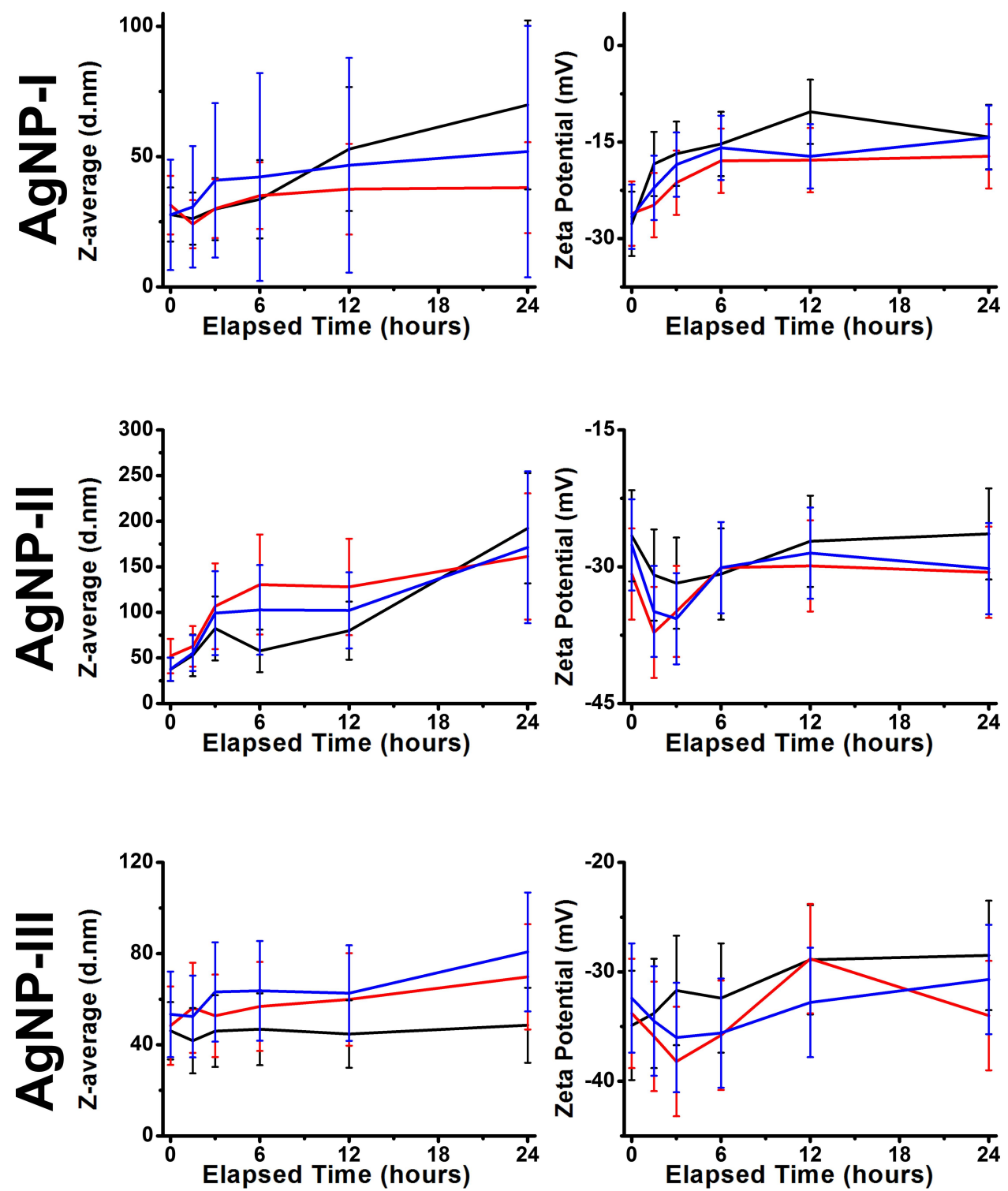

Figure 6 Dynamic light scattering results of citrate capped silver nanoparticle samples with increasing sizes (10 nm: AgNP-I, 20 nm: AgNP-II and 50 nm: AgNP-III) represented as average hydrodynamic diameter (Z-average) (right) and zeta potential (left) changes over 24 hours under external conditions with different glucose concentrations.

concentration, glucose could induce larger red shift after 1.5 hours compared to the control measurements by $\sim 40 \mathrm{~nm}$ in AgNP-II and $\sim 10 \mathrm{~nm}$ in AgNP-III, demonstrating the occurrence of surface interactions. Glutamine demonstrated similar tendencies, but the changes were less pronounced. Furthermore, it is also worth mentioning, that glutamine was able to decrease the absolute zeta potential of medium and large particles. However, as these zeta changes did not seem to affect aggregation grade, it can be speculated that even small biomolecules like glutamine can provide some degree of steric repulsion among particles.

In conclusion, small biomolecules like glucose and glutamine did not affect colloidal stability in the measured concentrations: although they affected zeta-potential and 


\section{Glutamine Dependence: \\ DLS}
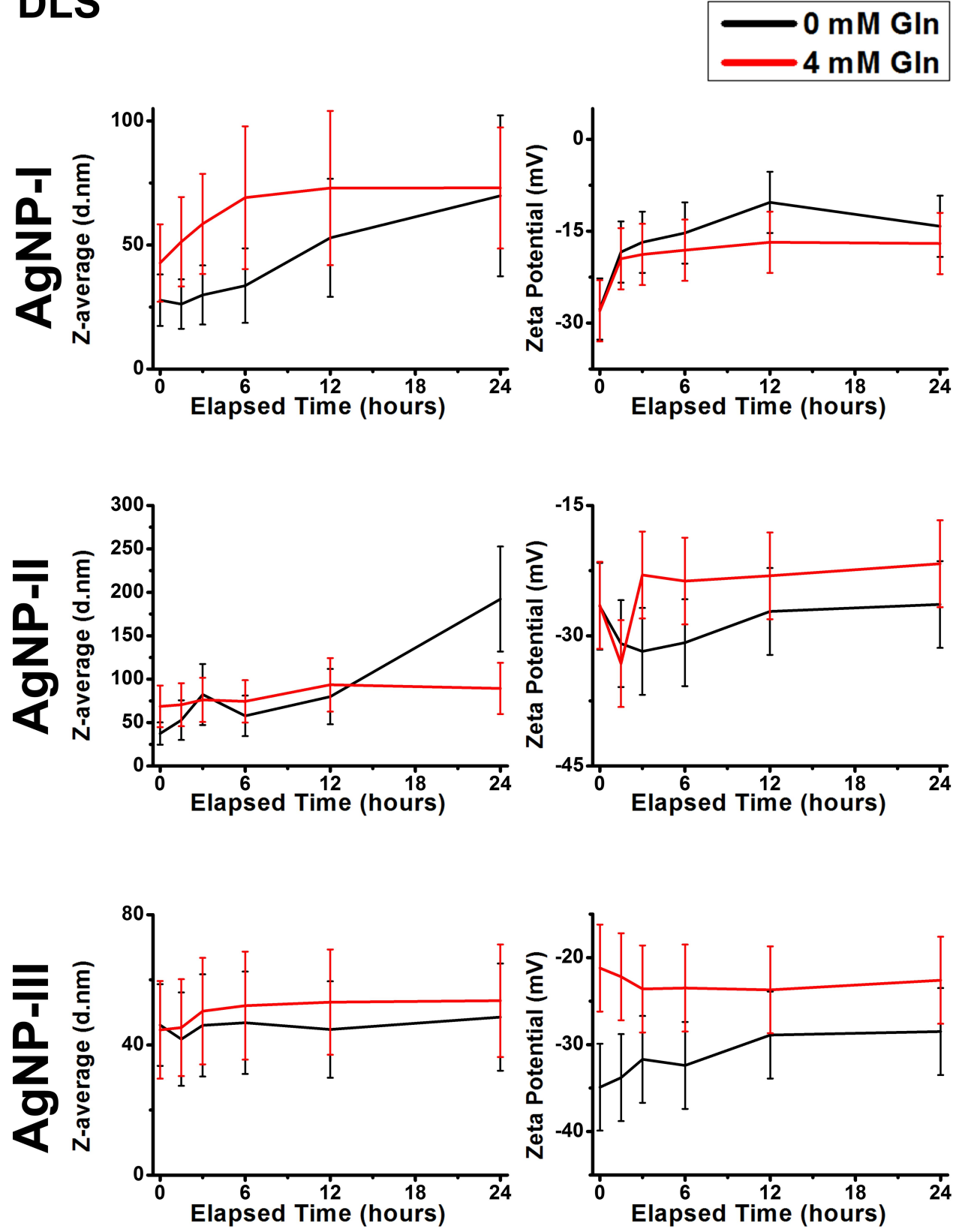

Figure 7 Dynamic light scattering results of citrate capped silver nanoparticle samples with increasing sizes (I0 nm: AgNP-I, $20 \mathrm{~nm}$ : AgNP-II and $50 \mathrm{~nm}$ : AgNP-III) represented as average hydrodynamic diameter (Z-average) (right) and zeta potential (left) changes over 24 hours in the presence of glutamine.

UV-Vis results to varying degrees, Z-average results did not coincide. This indicates the surface adsorption of the molecules, dampening electrostatic repulsion but at the same time providing steric stabilization.

\section{The Effect of Cell Culture Components}

To contextualize previous results and to model biological conditions more adeptly, some of the most often utilized cell culture components were selected and used as experimental conditions for investigating AgNP colloidal stability. Throughout in vitro experiments, one of the most important functions of DMEM as a culture medium is to establish necessary osmotic conditions, but from a chemical standpoint, it is a complex salt solution with a similar total ionic strength as $150 \mathrm{mM} \mathrm{NaCl}{ }^{40}$ As for FBS, it is an intricate mixture of biomolecules - mainly 
proteins - that were expected to show some similarities with the experimental results of glucose and glutamine from a surface adsorption standpoint, despite having a much more complex chemical composition and diversity. ${ }^{19}$ The DLS and UV-Vis results presented in Figure 8 and Supplementary Figure S5 respectively can be explained by examining the chemical composition of these materials and relate them to the measurements in the previous sections.

Diluting differently sized AgNPs in DMEM produced similar effects on colloidal stability as was observed in the presence of high $\mathrm{NaCl}$ concentration. AgNP dispersion in $50 \mathrm{v} / \mathrm{v} \%$ DMEM demonstrated large scale aggregation detected as the increase of zeta potentials and Z-average
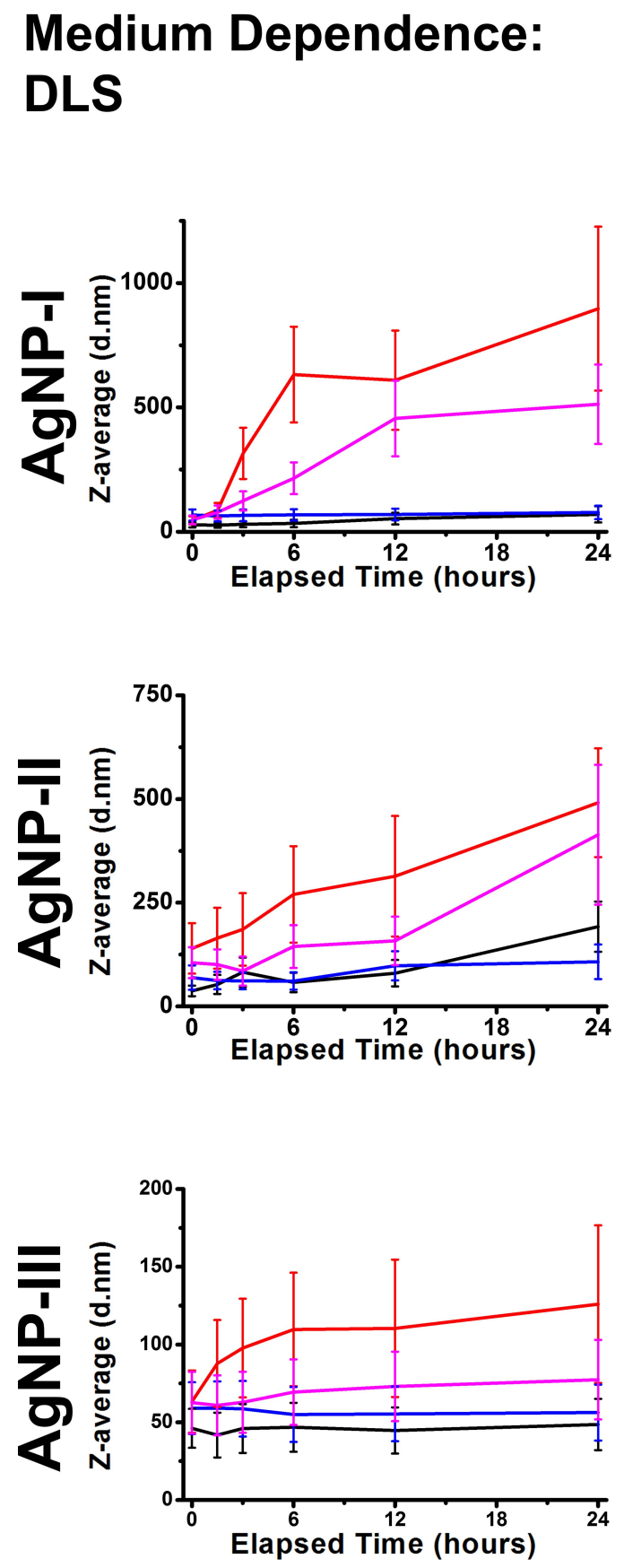
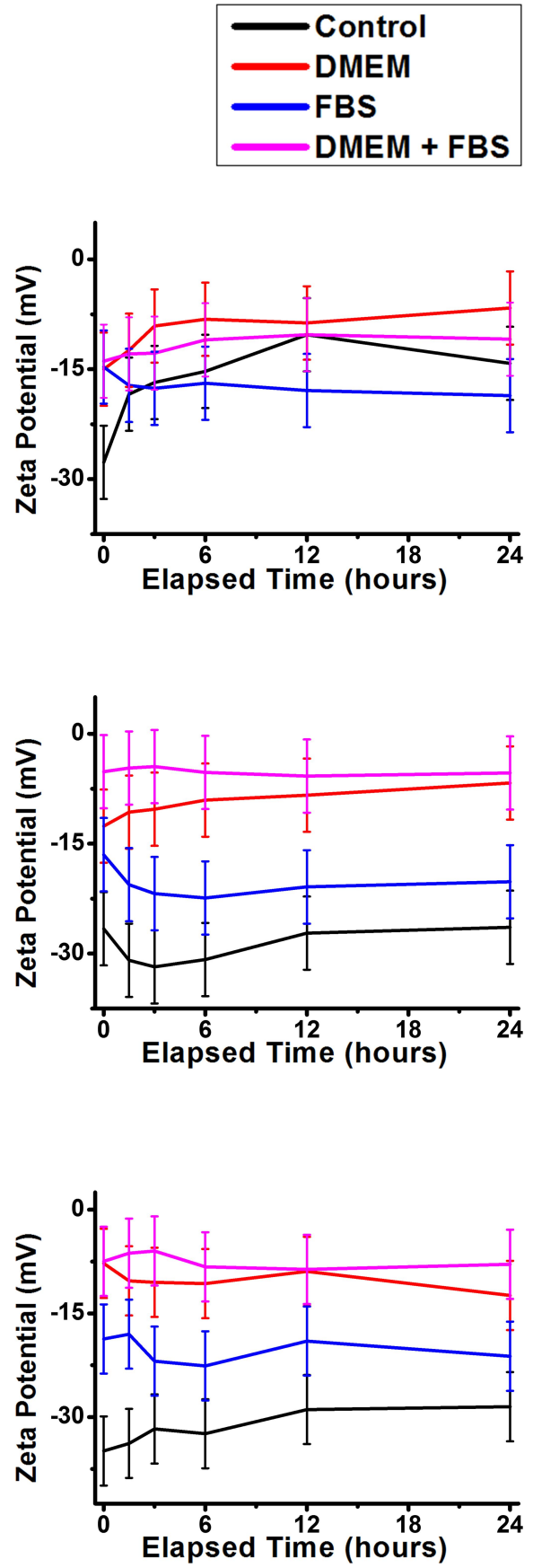

Figure 8 Dynamic light scattering results of citrate capped silver nanoparticle samples with increasing sizes (I0 nm: AgNP-I, $20 \mathrm{~nm}$ : AgNP-II and $50 \mathrm{~nm}$ : AgNP-III) represented as average hydrodynamic diameter (Z-average) (right) and zeta potential (left) changes over 24 hours in the presence of cell culture components DMEM and FBS. 
values, furthermore as steep declines in SPR intensity. It is interesting to note, that the maximum aggregate sizes induced by DMEM after 24 hours were inversely proportional to primer nanoparticle size.

FBS showed similar, but stronger interactions with AgNPs as those observed in the presence of smaller molecules such as glucose and glutamine. Z-average of the particles remained unaffected, while a zeta potential increase was detected. The SPR peaks demonstrated a slight redshift, but perhaps more interestingly, SPR intensities did not decline as noticeably as in the control measurements. These results can be explained by the innate adsorption of macromolecules on nanoparticle surfaces (bottom row of Figure 4), the phenomenon of which in vivo is now understood as biomolecular corona formation. $^{49}$

Perhaps the most important effect of corona formation within our experiments was its capacity to counteract aggregation to a degree (keeping Z-average in lower values) by administering electrosteric repulsion among particles, evidenced by the moderate increase of Z-average and mediated changes in SPR compared to the DMEM results. The combined strength of the inherent resistance of larger AgNPs against aggregation and biomolecular corona formation culminated in the revelation that in this seed-mediated growth system, in vitro colloidal stability was inversely proportional to nanoparticle size as, despite its largest size, AgNP-III produced the smallest and optically most active aggregates.

In summary, the in vitro cell culture components DMEM and FBS induced similar aggregation tendencies as the investigated simpler salt and biomolecule models respectively, emphasizing their chemical composition, DMEM being a salt-rich culture environment and FBS containing a wide variety of biomolecules (mostly proteins). ${ }^{40}$ Surface adsorption proved to be a defining characteristic of FBS: the resulting coating, commonly referred to as biomolecular corona, could counteract the severity of salt-induced aggregation in experiments containing both DMEM and FBS, in good agreement with other studies. $^{31,35,49}$

\section{Biological Analysis Cytotoxicity Assays}

In order to explore how the different aggregation states can affect the cytotoxicity of nanosilver, we performed twostep MTT cell viability assays on human prostate cancer cells (DU145) and on immortalized human keratinocyte cells $(\mathrm{HaCaT})$. For the investigation of the aggregationdependent toxicity, first, the viability of both cells was measured after AgNP-I, AgNP-II, and AgNP-III treatments, and the $\mathrm{IC}_{50}$ concentrations were determined. Thereafter, in the second step, to evaluate the changes in AgNP cytotoxicity caused by particle aggregation in a time-dependent manner, different silver nanoparticle aggregation states were formulated and the aggregationdependent toxicity of all three silver samples was examined at nanoparticle concentrations corresponding to the respective cell line-specific $\mathrm{IC}_{50}\left(\mathrm{IC}_{50}\right.$ values on $\mathrm{DU} 145$ cells: AgNP-I=8.28 ppm; AgNP-II=10.41 ppm; AgNP-III $=12.53 \mathrm{ppm} ; \mathrm{IC}_{50}$ values on HaCaT cells: AgNP-I=1.96 ppm; AgNP-II=3.08 ppm; AgNP-III=11.67 ppm). These MTT experiments were assessed by incubating the cells with aggregated AgNP samples for 24 hours. Nanoparticle aggregation states were created by adding $150 \mathrm{mM} \mathrm{NaCl}$ to silver samples for $0,1.5,3,6,12$, and 24 hours at $37^{\circ} \mathrm{C}$ prior to the beginning of the toxicity assays, as our preliminary results indicated that among the tested experimental conditions the electrolyte concentration had the greatest impact on nanoparticle aggregation.

The MTT viability assays in Figure 9 revealed that cell viability increased, and nanoparticle toxicity decreased with growing aggregation grade in case of all samples. For all three samples, the cell viability was the lowest $(\sim 50 \%)$ when cells were in contact with AgNPs without $\mathrm{NaCl}$ pre-incubation. The viability of both DU145 and HaCaT cells were higher when they were treated with AgNP samples, which were previously aggregated by incubation with $150 \mathrm{mM} \mathrm{NaCl}$. Cell viability increased with increasing aggregation time. In addition, particle sizedependent effect on cell viability could be observed, while AgNP-I exerted no toxicity after incubation with $\mathrm{NaCl}$, resulting in instantaneous toxicity loss against $\mathrm{HaCaT}$, and gradual loss by 24 hours against DU145 respectively. AgNP-II was more stable; it lost its effectiveness regarding HaCaT similarly to AgNP-I but retained mild toxicity against DU145 after incubation with $\mathrm{NaCl}$. Ultimately, AgNP-III could retain a small degree of cytotoxic activity for the entire aggregation timeframe $(\sim 60-80 \%$ cell viability).

\section{Antimicrobial Studies}

We also intended to assess the changes in the antimicrobial activity of differently sized AgNP colloids caused by particle aggregation. Therefore, the antibacterial and antifungal activities of the aggregated silver samples (AgNP-I, 


\section{AgNP-I}

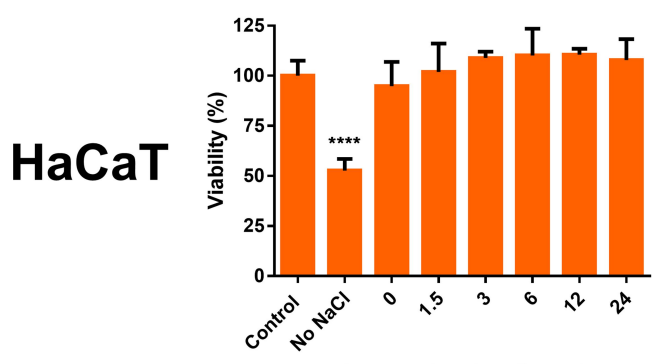

Aggregation time (hours)

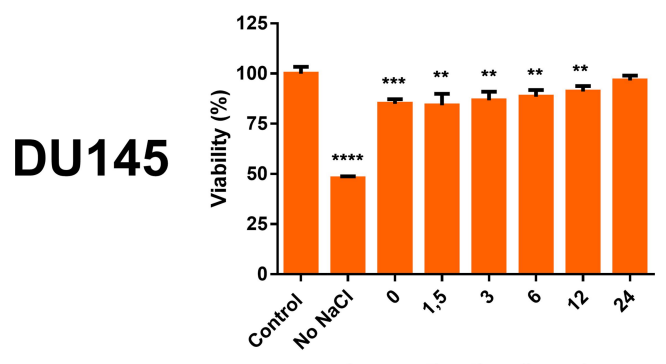

Aggregation time (hours)
AgNP-II

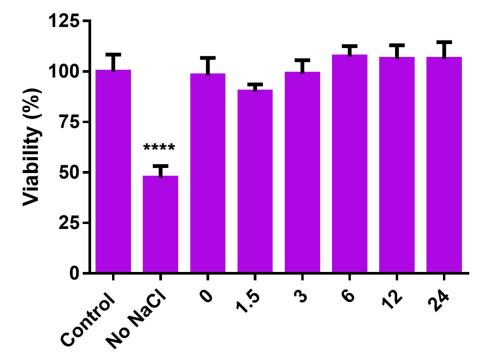

Aggregation time (hours)

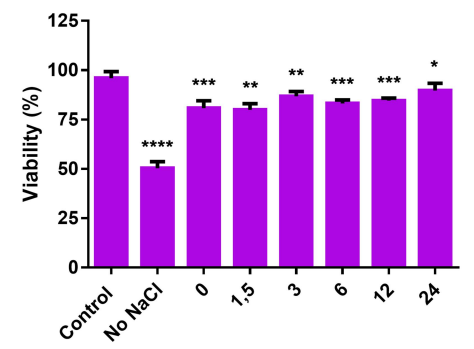

Aggregation time (hours)
AgNP-III

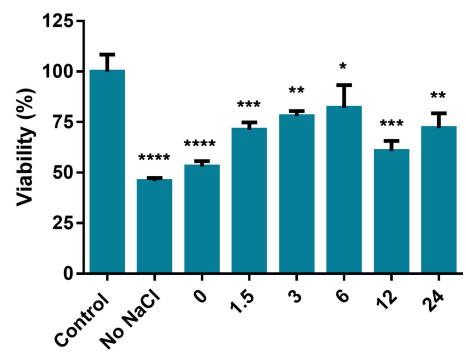

Aggregation time (hours)

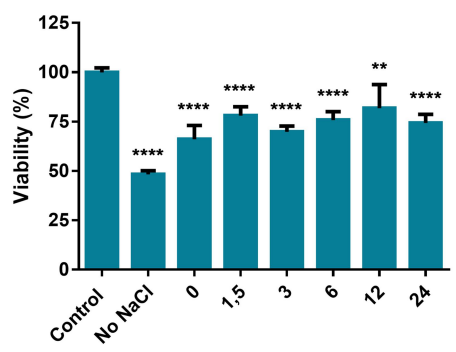

Aggregation time (hours)

Figure 9 The effect of nanoparticle aggregation (citrate capped silver nanoparticle samples with increasing sizes: $10 \mathrm{~nm}$ : AgNP-I (left), $20 \mathrm{~nm}$ : AgNP-II (middle) and $50 \mathrm{~nm}$ : AgNP-III (right)) on the cytotoxicity toward immortalized human keratinocyte cells ( $\mathrm{HaCaT}$ ) and human prostate cancer (DUI45) cells. Increasing aggregation states were created by incubating the particles with $150 \mathrm{mM} \mathrm{NaCl}$ for different time periods $(0,1.5,3,6,12$, and 24 hours) prior to cell treatments. The statistical significance of the experiments was calculated through unpaired $t$-tests and are marked with $*(\mathrm{p} \leq 0.05), * *(\mathrm{p} \leq 0.0 \mathrm{I})$, ***(p $\leq 0.00 \mathrm{I})$ and $* * * *(\mathrm{p} \leq 0.000 \mathrm{I})$.

AgNP-II, and AgNP-III) were tested against Bacillus megaterium, Escherichia coli, and Cryptococcus neoformans strains using the same system as for the cytotoxicity tests, with the exception that instead of $\mathrm{IC}_{50}$ concentrations, the corresponding minimal inhibitory concentrations (MIC) were defined first, and then used throughout the microbiology experiments.

The minimal inhibitory concentration of AgNP-I was 18.75 ppm for C. neoformans and B. megaterium, and 37.5 ppm for $E$. coli, as published in our paper previously. ${ }^{7}$ The MIC values for AgNP-II and AgNP-III were lower than those of AgNP-I, against C. neoformans (15.63 ppm) and B. megaterium (7.81 ppm), but were higher (62.5 ppm) for E. coli.

We found that the inhibitory effect of the aggregated nanoparticles decreased with growing nanoparticle aggregation grade (Figure 10) throughout the experiments, in accordance with the cytotoxicity results. A similar size-dependent activity - differences between the effects of the three differently sized AgNP samples - could also be observed. Microbial cell viability was the lowest when cells were exposed to AgNPs which were previously not aggregated, while the viability of all three strains increased when they were treated with AgNP samples which were at different aggregation grades due to incubation with $\mathrm{NaCl}$ for different time periods.

As previously reported, AgNP-I was non-toxic after 6 hours' incubation with $\mathrm{NaCl}$ for B. megaterium and E. coli while its effectiveness against $C$. neoformans was lost after 24 hours of aggregation with $\mathrm{NaCl}$. In the case of AgNPII, no toxicity was detected after 24- and 6-hours' incubation with $\mathrm{NaCl}$ for $B$. megaterium and $E$. coli, respectively. ${ }^{7}$ While the toxicity to $C$. neoformans was not affected by aggregation after 3 hours, the particles were approximately $45 \%$ effective.

In line with the human toxicity data, we found that AgNP-III could retain a certain degree of inhibitory activity for the entire aggregation timespan $(\sim 60 \%$ effectiveness for B. megaterium, $\sim 70 \%$ for E. coli, and $\sim 50 \%$ for C. neoformans).

The comprehensive in vitro analysis verified that aggregation should indeed be taken into consideration in association with nanotherapeutic applications. While the actual degrees might vary, toxicity reduction due to 


\section{AgNP-I}
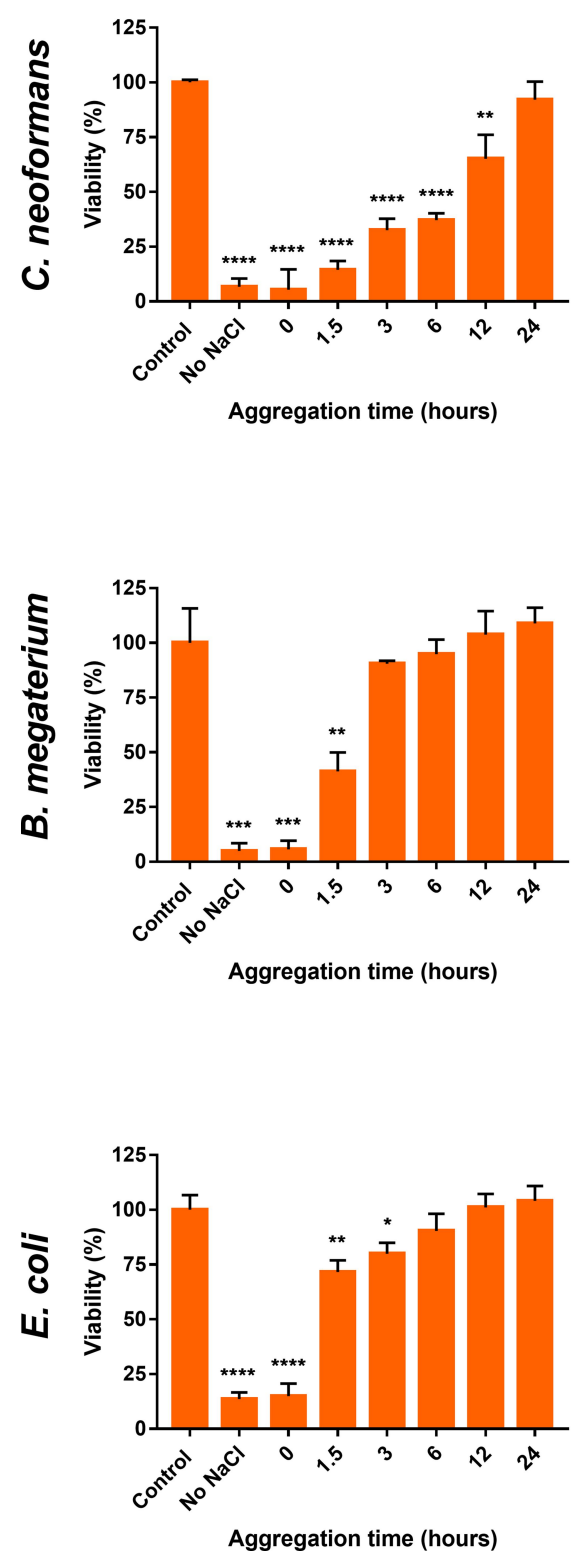

AgNP-II

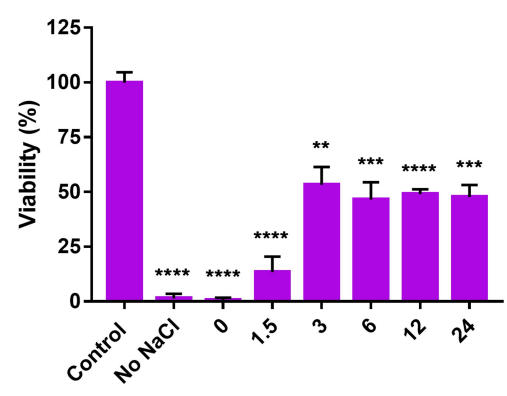

Aggregation time (hours)

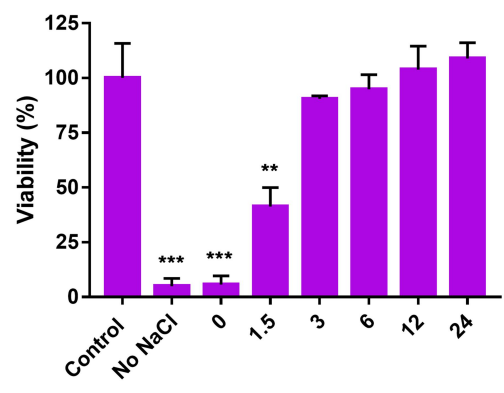

Aggregation time (hours)

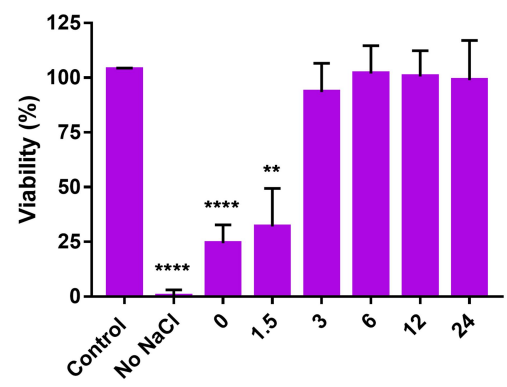

Aggregation time (hours)

\section{AgNP-III}

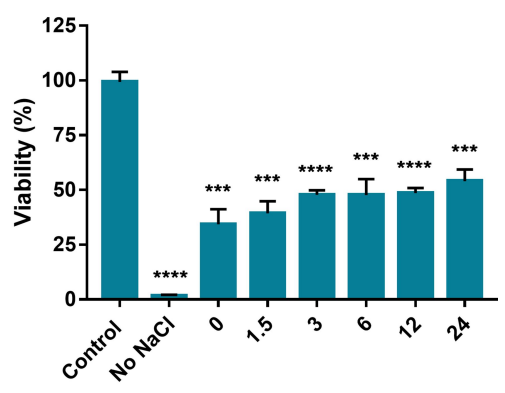

Aggregation time (hours)

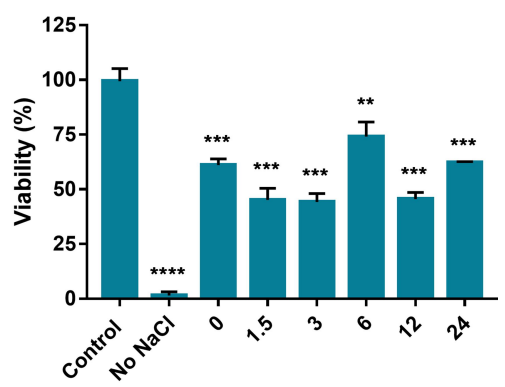

Aggregation time (hours)

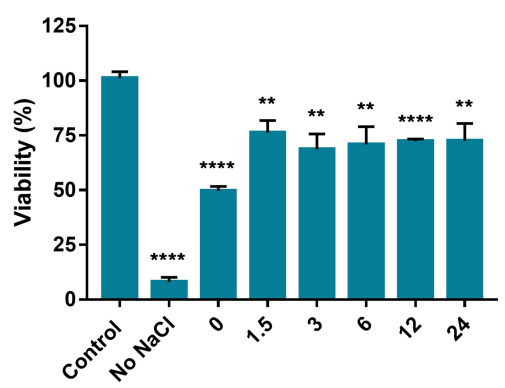

Aggregation time (hours)

Figure 10 The effect of nanoparticle aggregation (citrate capped silver nanoparticle samples with increasing sizes: $10 \mathrm{~nm}$ : AgNP-I (I.row), $20 \mathrm{~nm}$ : AgNP-II (2.row) and 50 $\mathrm{nm}$ : AgNP-III (3.row)) on the antimicrobial activity against $C$. neoformans, B. megaterium, and $E$. coli. Increasing aggregation states were created by incubating the particles with $150 \mathrm{mM} \mathrm{NaCl}$ for different time periods $(0,1.5,3,6,12$, and 24 hours) prior to cell treatments. The statistical significance of the experiments was calculated through unpaired $t$-tests and are marked with $*(p \leq 0.05), * *(p \leq 0.01), * * *(p \leq 0.00 I)$ and $* * * *(p \leq 0.000 I)$.

aggregation was a universal phenomenon throughout the experiments. Furthermore, the nanoparticle size-dependent resistance toward aggregation concluded from the DLS and UV-Vis measurements was demonstrated to have a biological effect as well.

Comparing the results on differently sized AgNPs, an interesting conclusion can be drawn: increasing particle size decreases the AgNP sensitivity toward aggregation induced by external factors. This is essential information for the rational design of biologically active nanoparticles aimed at biomedical applications. Our experimental results imply that various biorelevant conditions affect silver nanoparticle aggregation, and this propensity influences their biological activity massively. It can be stated, that 
increasing nanoparticle size can substantially alleviate the severity of aggregation that will inevitably occur in a biological system, or a natural environment. This conclusion contests the current general approach of the relevant literature where the concept "smaller is always better" is promoted when it comes to the biological application of nanomaterials. While it is well documented that smaller nanoparticles of the same bioactive chemical composition possess stronger toxicity, our findings indicate that a balance must be established between toxicity and longevity, should we desire lasting biological effects. This trade-off could have serious implications on how we look at nanoparticle toxicity in scientific research.

\section{Conclusion}

In this study, three AgNP samples containing nanoparticles in increasing diameters were prepared by seed-mediated growth. After their successful material scientific characterization, aggregation behavior measurements were performed on all three samples to investigate their colloidal stability in lifelike environments. Acidic pH and physiological salt concentration proved to have a substantial effect on AgNP aggregation, while biomolecules - from simple compounds like glucose and glutamine, to complex macromolecules like blood plasma proteins - demonstrated a tendency for surface adsorption which led to biomolecular corona formation. Cytotoxicity and antimicrobial studies proved that aggregation can attenuate, or in extreme cases even completely abolish AgNP bioactivity. Therefore, biomolecular corona formation proved to be a fundamental phenomenon, as it could counteract aggregation induced by outside conditions, protecting toxicity.

While our observations demonstrated universal tendencies for all three AgNP samples, particle size proved to be a defining characteristic affecting biological nanoparticle utilizations yet again, however, in an opposing way to what can usually be seen in the relevant literature. In most cases, synthetic approaches are aimed at creating particles with as small diameters as possible, since those possess the highest toxicity. Our results on the other hand proved that although that observation is true, increasing NP size increases colloidal stability and also provides greater strength against conditions in the close surroundings of the particles affecting aggregation.

Should our findings prove to have relevance outside this experimental system, an interesting dilemma is introduced that has to be tackled when nanomaterials are used for biological applications: if smaller particles promise greater biological activity, yet in such conditions, they become ineffective in a few hours, while larger, less active materials might have a lasting effect, just how small should we be aiming for?

\section{Acknowledgments}

The work was supported by the ÚNKP-20-4-SZTE-580 (P. B.) and the ÚNKP-20-5-SZTE-655 (M.K.) New National Excellence Program of the Ministry for Innovation and Technology from the source of the National Research, Development, and Innovation Fund, and by the János Bolyai Research Scholarship of the Hungarian Academy of Sciences (BO/00878/19/8 for M.K.). Financial support by the National Research, Development, and Innovation Office-NKFIH through projects GINOP-2.3.2-15-201600038 and GINOP-2.3.2-15-2016-00035 is gratefully acknowledged.

\section{Disclosure}

The authors report no conflicts of interest in this work.

\section{References}

1. Pryshchepa O, Pomastowski P, Buszewski B. Silver nanoparticles: synthesis, investigation techniques, and properties. Adv Colloid Interface Sci. 2020;284:102246. doi:10.1016/j.cis.2020.102246

2. Ivask A, Kurvet I, Kasemets K, et al. Size-dependent toxicity of silver nanoparticles to bacteria, yeast, algae, crustaceans and mammalian cells in vitro. PLoS One. 2014;9(7):e102108. doi:10.1371/journal. pone. 0102108

3. Kovács D, Igaz N, Keskeny $\mathrm{C}$, et al. Silver nanoparticles defeat p53-positive and p53-negative osteosarcoma cells by triggering mitochondrial stress and apoptosis. Sci Rep. 2016;6(1):27902. doi:10.1038/ srep27902

4. Gopisetty MK, Kovács D, Igaz N, et al. Endoplasmic reticulum stress: major player in size-dependent inhibition of P-glycoprotein by silver nanoparticles in multidrug-resistant breast cancer cells. J Nanobiotechnology. 2019;17(1):9. doi:10.1186/s12951-019-0448-4

5. Szerencsés B, Igaz N, Tóbiás Á, et al. Size-dependent activity of silver nanoparticles on the morphological switch and biofilm formation of opportunistic pathogenic yeasts. BMC Microbiol. 2020;20(176):13. doi:10.1186/s12866-020-01858-9

6. Wan Y, Guo Z, Jiang X, et al. Quasi-spherical silver nanoparticles: aqueous synthesis and size control by the seed-mediated Lee-Meisel method. J Colloid Interface Sci. 2013;394:263-268. doi:10.1016/j. jcis.2012.12.037

7. Shrivas K, Nirmalkar N, Ghosale A, Thakur SS, Shankar R. Enhancement of plasmonic resonance through an exchange reaction on the surface of silver nanoparticles: application to the highly selective detection of triazophos pesticide in food and vegetable samples. RSC Adv. 2016;6(84):80739-80747. doi:10.1039/c6ra16097b

8. Wulandari P, Nagahiro T, Fukada N, Kimura Y, Niwano M, Tamada K. Characterization of citrates on gold and silver nanoparticles. J Colloid Interface Sci. 2015;438:244-248. doi:10.1016/j.jcis.2014.09.078

9. Rónavári A, Kovács $\mathrm{D}$, Igaz $\mathrm{N}$, et al. Biological activity of green-synthesized silver nanoparticles depends on the applied natural extracts: a comprehensive study. Int J Nanomedicine. 2017; Volume 12:871-883. doi:10.2147/IJN.S122842 
10. El Badawy AM, Scheckel KG, Suidan M, Tolaymat T. The impact of stabilization mechanism on the aggregation kinetics of silver nanoparticles. Sci Total Environ. 2012;429:325-331. doi:10.1016/j. scitotenv.2012.03.041

11. Qin D, Yang G, Wang Y, Zhou Y, Zhang L. Green synthesis of biocompatible trypsin-conjugated Ag nanocomposite with antibacterial activity. Appl Surf Sci. 2019;469:528-536. doi:10.1016/j. apsusc.2018.11.057

12. Song Z, Wu Y, Wang H, Han H. Synergistic antibacterial effects of curcumin modified silver nanoparticles through ROS-mediated pathways. Mater Sci Eng C. 2019;99:255-263. doi:10.1016/j. msec.2018.12.053

13. Tsuda A, Konduru NV. The role of natural processes and surface energy of inhaled engineered nanoparticles on aggregation and corona formation. NanoImpact. 2016;2:38-44. doi:10.1016/j. impact.2016.06.002

14. Hotze EM, Phenrat T, Lowry GV. Nanoparticle aggregation: challenges to understanding transport and reactivity in the environment J Environ Qual. 2010;39(6):1909-1924. doi:10.2134/jeq2009.0462

15. Badawy AME, Luxton TP, Silva RG, Scheckel KG, Suidan MT, Tolaymat TM. Impact of environmental conditions $(\mathrm{pH}$, Ionic Strength, and Electrolyte Type) on the surface charge and aggregation of silver nanoparticles suspensions. Environ Sci Technol. 2010;44 (4):1260-1266. doi:10.1021/es902240k

16. Huynh KA, Chen KL. Aggregation kinetics of citrate and polyvinylpyrrolidone coated silver nanoparticles in monovalent and divalent electrolyte solutions. Environ Sci Technol. 2011;45(13):5564-5571. doi:10.1021/es200157h

17. Ravindran A, Singh A, Raichur AM, Chandrasekaran N, Mukherjee A. Studies on interaction of colloidal Ag nanoparticles with Bovine Serum Albumin (BSA). Colloids Surf B Biointerfaces. 2010;76(1):32-37. doi:10.1016/J.COLSURFB.2009.10.005

18. Radomski A, Jurasz P, Alonso-Escolano D, et al. Nanoparticleinduced platelet aggregation and vascular thrombosis. $\mathrm{Br}$ J Pharmacol. 2005;146(6):882-893. doi:10.1038/sj.bjp.0706386

19. Bélteky P, Rónavári A, Igaz N, et al. Silver nanoparticles: aggregation behavior in biorelevant conditions and its impact on biological activity. Int J Nanomedicine. 2019; Volume 14:667-687. doi:10.2147/ IJN.S185965

20. Panáček A, Kvítek L, Smékalová M, et al. Bacterial resistance to silver nanoparticles and how to overcome it. Nat Nanotechnol. 2018;13(1):65-71. doi:10.1038/s41565-017-0013-y

21. Gordon O, Slenters TV, Brunetto PS, et al. Silver coordination polymers for prevention of implant infection: thiol interaction, impact on respiratory chain enzymes, and hydroxyl radical induction. Antimicrob Agents Chemother. 2010;54(10):4208-4218. doi:10.1128/AAC.01830-09

22. Park HJ, Kim JY, Kim J, et al. Silver-ion-mediated reactive oxygen species generation affecting bactericidal activity. Water Res. 2009;43 (4):1027-1032. doi:10.1016/j.watres.2008.12.002

23. Dibrov P, Dzioba J, Gosink KK, Häse CC. Chemiosmotic mechanism of antimicrobial activity of $\mathrm{Ag}+$ in vibrio cholerae. Antimicrob Agents Chemother. 2002;46(8):2668-2670. doi:10.1128/ AAC.46.8.2668-2670.2002

24. Reidy B, Haase A, Luch A, Dawson K, Lynch I. Mechanisms of silver nanoparticle release, transformation and toxicity: a critical review of current knowledge and recommendations for future studies and applications. Materials (Basel). 2013;6(6):2295-2350. doi:10.3390/ma6062295

25. Sun X, Shi J, Zou X, Wang C, Yang Y, Zhang H. Silver nanoparticles interact with the cell membrane and increase endothelial permeability by promoting VE-cadherin internalization. $J$ Hazard Mater. 2016;317:570-578. doi:10.1016/j.jhazmat.2016.06.023

26. Teodoro JS, Simões AM, Duarte FV, et al. Assessment of the toxicity of silver nanoparticles in vitro: a mitochondrial perspective. Toxicol Vitr. 2011;25(3):664-670. doi:10.1016/j.tiv.2011.01.004
27. AshaRani PV, Hande MP, Valiyaveettil S. Anti-proliferative activity of silver nanoparticles. BMC Cell Biol. 2009;10(1):65. doi:10.1186/ 1471-2121-10-65

28. Bhattacharjee S. DLS and zeta potential - what they are and what they are not? J Control Release. 2016;235:337-351. doi:10.1016/j. jconrel.2016.06.017

29. García MA. Surface Plasmons in metallic nanoparticles: fundamentals and applications. J Phys D Appl Phys. 2012;44(28). doi:10.1088/ 0022-3727/45/38/38950

30. Huang T, Xu XN. Synthesis and characterization of tunable rainbow colored colloidal silver nanoparticles using single-nanoparticle plasmonic microscopy and spectroscopy. J Mater Chem. 2010;20 (44):9867-9876. doi:10.1039/c0jm01990a

31. Levak M, Burić P, Dutour Sikirić M, et al. Effect of protein corona on silver nanoparticle stabilization and ion release kinetics in artificial seawater. Environ Sci Technol. 2017;51(3):1259-1266. doi:10.1021/ acs.est.6b03161

32. Niu W, Zhang L, Xu G. Seed-mediated growth of noble metal nanocrystals: crystal growth and shape control. Nanoscale. 2013;5 (8):3172-3181. doi:10.1039/c3nr00219e

33. Schneider CA, Rasband WS, Eliceiri KW. NIH Image to ImageJ: 25 years of image analysis. Nat Methods. 2012;9(7):671-675. doi:10.1038/nmeth.2089

34. Schindelin J, Arganda-Carreras I, Frise E, et al. Fiji: an open-source platform for biological-image analysis. Nat Methods. 2012;9 (7):676-682. doi:10.1038/nmeth.2019

35. Alarcon EI, Bueno-Alejo CJ, Noel CW, et al. Human serum albumin as protecting agent of silver nanoparticles: role of the protein conformation and amine groups in the nanoparticle stabilization. $J$ Nanoparticle Res. 2013;15(1):1374. doi:10.1007/s11051-0121374-7

36. Szekeres GP, Kneipp J. SERS probing of proteins in gold nanoparticle agglomerates. Front Chem. 2019;7:30. doi:10.3389/ fchem.2019.00030

37. Ghosh SK, Pal T. Interparticle coupling effect on the surface plasmon resonance of gold nanoparticles: from theory to applications. Chem Rev. 2007;107(11):4797-4862. doi:10.1021/cr0680282

38. Kararli TT. Comparison of the gastrointestinal anatomy, physiology, and biochemistry of humans and commonly used laboratory animals. Biopharm Drug Dispos. 1995;16(5):351-380. doi:10.1002/ bdd. 2510160502

39. Eichelberger L, Richter RB. Water, nitroden and electrolyte concentration in the brain. $J$ Biol Chem. 1944;154(1):21-29. doi:10.1016/ S0021-9258(18)71938-8

40. Yao T, Asayama Y. Animal-cell culture media: history, characteristics, and current issues. Reprod Med Biol. 2017;16(2):99-117. doi: $10.1002 / \mathrm{rmb} 2.12024$

41. Freckmann G, Hagenlocher S, Baumstark A, et al. Continuous glucose profiles in healthy subjects under everyday life conditions and after different meals. J Diabetes Sci Technol. 2007;1(5):695-703. doi:10.1177/193229680700100513

42. Strazzullo P, Leclercq C. Sodium. Adv Nutr. 2014;5(2):188-190. doi:10.3945/an.113.005215

43. Wagner T, Lipinski H-G. IJBlob: an ImageJ library for connected component analysis and shape analysis. J Open Res Softw. 2013;1(1): e6. doi:10.5334/jors.ae

44. Che Q, Yang H, Lu L, Wang Y. Nanoparticles-aided silver front contact paste for crystalline silicon solar cells. J Mater Sci Mater Electron. 2013;24(2):524-528. doi:10.1007/s10854-012-0941-0

45. Pal S, Tak YK, Song JM. Does the antibacterial activity of silver nanoparticles depend on the shape of the nanoparticle? A study of the gram-negative bacterium Escherichia coli. Appl Environ Microbiol. 2007;73(6):1712-1720. doi:10.1128/AEM.02218-06

46. Xu R, Wang D, Zhang J, Li Y. Shape-dependent catalytic activity of silver nanoparticles for the oxidation of styrene. Chem Asian J. 2006;1(6):888-893. doi:10.1002/asia.200600260 
47. He YT, Wan J, Tokunaga T. Kinetic stability of hematite nanoparticles: the effect of particle sizes. J Nanoparticle Res. 2008;10 (2):321-332. doi:10.1007/s11051-007-9255-1

48. He D, Bligh MW, Waite TD. Effects of aggregate structure on the dissolution kinetics of citrate-stabilized silver nanoparticles. Environ Sci Technol. 2013;47(16):9148-9156. doi:10.1021/es400391a
49. Hadjidemetriou M, Kostarelos K. Evolution of the nanoparticle corona. Nat Nanotechnol. 2017;12(4):288-290. doi:10.1038/ nnano.2017.61

\section{Publish your work in this journal}

The International Journal of Nanomedicine is an international, peerreviewed journal focusing on the application of nanotechnology in diagnostics, therapeutics, and drug delivery systems throughout the biomedical field. This journal is indexed on PubMed Central, MedLine, CAS, SciSearch ${ }^{\mathbb{R}}$, Current Contents ${ }^{\mathbb{R}} /$ Clinical Medicine, $^{2}$
Journal Citation Reports/Science Edition, EMBase, Scopus and the Elsevier Bibliographic databases. The manuscript management system is completely online and includes a very quick and fair peer-review system, which is all easy to use. Visit http://www.dovepress.com/ testimonials.php to read real quotes from published authors. 